\title{
Impact of spatial proximity on territoriality among human
} skin bacteria

\author{
Jhonatan A. Hernandez-Valdes (D) ${ }^{1}$, Lu Zhou ${ }^{1}$, Marcel P. de Vries ${ }^{2}$ and Oscar P. Kuipers $\mathbb{D}^{1 凶}$
}

Bacteria display social behavior and establish cooperative or competitive interactions in the niches they occupy. The human skin is a densely populated environment where many bacterial species live. Thus, bacterial inhabitants are expected to find a balance in these interactions, which eventually defines their spatial distribution and the composition of our skin microbiota. Unraveling the physiological basis of the interactions between bacterial species in organized environments requires reductionist analyses using functionally relevant species. Here, we study the interaction between two members of our skin microbiota, Bacillus subtilis and Staphylococcus epidermidis. We show that $B$. subtilis actively responds to the presence of $S$. epidermidis in its proximity by two strategies: antimicrobial production and development of a subpopulation with migratory response. The initial response of $B$. subtilis is production of chlorotetain, which degrades the $S$. epidermidis at the colony level. Next, a subpopulation of $B$. subtilis motile cells emerges. Remarkably this subpopulation slides towards the remaining $S$. epidermidis colony and engulfs it. A slow response back from $S$. epidermidis cells give origin to resistant cells that prevent both attacks from $B$. subtilis. We hypothesized that this niche conquering and back-down response from $B$. subtilis and $S$. epidermidis, respectively, which resembles other conflicts in nature as the ones observed in animals, may play a role in defining the presence of certain bacterial species in the specific microenvironments that these bacteria occupy on our skin.

npj Biofilms and Microbiomes (2020)6:30; https://doi.org/10.1038/s41522-020-00140-0

\section{INTRODUCTION}

Diverse populations of human-associated bacteria live on the skin and are harmless or beneficial to their human host ${ }^{1}$ Symbiotic bacteria occupy skin niches and protect against colonization by pathogenic bacteria ${ }^{2}$. For example, Bacillus subtilis is known to protect the skin by producing bacitracin, a compound that inhibits the growth of other bacteria ${ }^{3,4}$. Although extensive research on the influence of the skin bacteria in the skin health has been performed ${ }^{5,6}$, few studies have investigated the specific interactions between members of the skin microbiota. The elucidation of these interactions is necessary to understand the complex organization of microbial communities e.g., the human microbiota.

Since the skin surface is a nutrient-poor niche ${ }^{1}$, microbial species are likely to compete for resources and colonization. The outcome of this microbial competition is the delicate balance in the diversity of bacterial species that inhabit our skin ${ }^{7,8}$. Bacteria employ different chemical and physical mechanisms to harm, inhibit or kill their competitors ${ }^{9}$. Chemical mechanisms include secretion of broad-spectrum antibiotics ${ }^{10}$ or strain-specific bacteriocins ${ }^{11}$, whereas physical mechanisms such as adhesion ${ }^{12}$ or secretion of polymeric substances ${ }^{13}$ allow bacteria to interfere with the growth of others. Here we study the potential interactions among Bacillus subtilis and Staphylococcus epidermidis. Both bacterial species are inhabitants of the same ecological niche, the human skin.

$B$. subtilis is a relevant member of the human microbiota. This bacterium produces and secretes several molecules to control the growth of other bacteria ${ }^{14}$, probably as an adaptive strategy to subsist in the densely populated environments where it is found (skin, digestive tract, extremities of human body and soil) ${ }^{7,15,16}$.
B. subtilis is the second most frequently associated bacteria in neonates ${ }^{17}$. In addition, in healthy individuals (including adults) it has been found in the outer ear $\operatorname{skin}^{17}$, and it is predominately found in the plantar skin of humans with strong foot odor ${ }^{18}$. S. epidermidis is a major inhabitant of the skin, and comprises more than $90 \%$ of the aerobic resident microbiota ${ }^{2}$. Despite the innocuous nature of $S$. epidermidis, this bacterium is currently seen as an important opportunistic pathogen in patients receiving medical devices ${ }^{19}$. S. epidermidis is one of the most frequent nosocomial infections, and in particular it is involved in the infection of catheters and implants ${ }^{19,20}$.

Recent studies show that bacteria form biofilms in response to ecological competition ${ }^{21}$. In this respect, Bacillus subtilis is a master in cell differentiation and several subpopulations of functionally distinct cell types coexist within its biofilms ${ }^{22}$. Besides biofilm formation, $B$. subtilis is a model for motility of Gram-positive bacteria; it is able to swim, swarm and slide ${ }^{23,24}$. The use of flagella allows the bacterium to swim and swarm, but the flagellumindependent sliding occurs due to growth ${ }^{25}$. Sliding is a poorly understood process, but it is known to depend upon multiple factors, such as the production of surfactin and/or the extracellular proteins BsIA and Tas $\mathrm{A}^{25-27}$.

Here, we study the $B$. subtilis antimicrobial production and sliding motility in response to the presence of $S$. epidermidis. Bacterial colonies are able to interact at a close proximity, and by using a colony model we simulate the interactions between bacterial communities. Firstly, we start our analysis by visualizing the $B$. subtilis production of antimicrobials, which inhibit the growth of S. epidermidis. Cells of the attacked S. epidermidis colony rapidly develop resistance and give rise to resistant colonies. The traditional approach to study antimicrobials is based on the

\footnotetext{
'Department of Molecular Genetics, Groningen Biomolecular Sciences and Biotechnology Institute, University of Groningen, Nijenborgh 7, 9747 AG Groningen, The Netherlands. ${ }^{2}$ Department of Biomedical Engineering Antonius Deusinglaan 1, University Medical Center Groningen, Groningen University, 9713 AW Groningen, Netherlands. ${ }^{\times}$email: o.p.kuipers@rug.nl
} 
extraction of the compounds from culture supernatants, followed by screening of fractions to identify the inhibitory molecules ${ }^{28}$. Bacterial competition approaches, where two bacteria are cocultured, allow us to revisit the mechanisms involved in competitive interactions between bacteria ${ }^{29}$. In this respect, genomic DNA sequencing of $S$. epidermidis resistant colonies revealed a point-nonsense mutation in the gene encoding the dipeptide transport system (DtpT) and several mutations in the gene encoding the biofilm-associated protein (Bap). Interestingly, bacilysin, the simplest peptide antibiotic known, is such a dipeptide synthesized by $B$. subtilis ${ }^{30}$. We demonstrate that $B$. subtilis produces chlorotetain, a halogenated variant of bacilysin, which kills S. epidermidis cells. Secondly, after the chemical attack, motile $B$. subtilis emerges from the colony to dramatically engulf the wild-type $S$. epidermidis colony. This migratory response is not developed towards the $S$. epidermidis resistant colonies. Therefore, our results suggest that functional Bap proteins indirectly trigger the motility of $B$. subtilis. Lastly, we show that the response regulator DegU regulates the behavior phenomena exhibited by $B$. subtilis, and it appears to mediate the interaction between $B$. subtilis and $\mathrm{S}$. epidermidis.

\section{RESULTS}

Interaction between $B$. subtilis and $S$. epidermidis colonies

We start by analyzing whether B. subtilis and S. epidermidis are able to interact at the colony level, using a chemically defined medium (CDM) to simulate the interactions among bacterial communities in poor-nutrient niches. To assess the interaction between colonies, both bacteria are spotted on a CDM-agar plate at different separation distances. This interaction assay shows that the colonies are able to interact at a close proximity, resulting in growth inhibition of S. epidermidis (Fig. 1). The shorter the distance between colonies, the higher the growth inhibition effect on the $S$. epidermidis colony. Notably, B. subtilis develops an active migratory response towards $S$. epidermidis. At the closest proximity, $B$. subtilis cells are able to engulf the colony of $S$. epidermidis. The growth inhibition and migratory response phenomena are not observed when each species is independently grown (Fig. S1). Moreover, we tested the interaction between $B$. subtilis with another skin bacterium (Cutibacterium brevis) and with other Gram-positive bacteria (Lactococcus lactis and Streptococcus thermophilus), and noted that the colony degradation only occurred against $S$. epidermidis (Fig. S2a). We observed the colony engulfment capacity of $B$. subtilis to other bacteria, as reported in a previous study on the interactions between different Bacillus species ${ }^{31}$. Interestingly, in an interaction assay between B. subtilis, S. epidermidis and L. lactis (Fig. S2b), $B$. subtilis specifically responds only to the presence of $S$. epidermidis. This result suggests that only $B$. subtilis and $S$. epidermidis appear to establish this specific interaction (antimicrobial production and motility) at the colony level.

Next, we examine the time it takes to observe both responses. To distinguish the growth of $B$. subtilis from $S$. epidermidis, we used a green fluorescent protein (GFP)-marked $B$. subtilis strain. Figure $1 \mathrm{~b}$ shows that the inhibitory growth effect occurs rapidly and is clearly visible at the end of $24 \mathrm{~h}$ of incubation, whereas the $B$. subtilis motility is a progressive response completed after 48 to $72 \mathrm{~h}$ of incubation. To this end, we aimed to distinguish the $B$. subtilis motility strategy. Swimming, swarming and sliding are the three motility strategies employed by $B$. subtilis ${ }^{32}$. Since an agar concentration below $1.5 \%(\mathrm{w} / \mathrm{v})$ in CDM-agar plates allow $B$. subtilis to swim and swarm, Fig. 1c shows that $48 \mathrm{~h}$ of incubation, $B$. subtilis is able to display motility towards $S$. epidermidis at even an agar concentration of $2 \%(\mathrm{w} / \mathrm{v})$. With respect to the growth inhibition effect on S. epidermidis, the varying concentrations of agar in the growth medium did not change the inhibitory effect.
These findings suggest that $B$. subtilis is able to attack $S$. epidermidis in two phases, the first phase involves antimicrobial production, and the second phase consists of mobilization of cells via sliding to engulf the neighbor colony.

\section{Genetic elements involved in the $B$. subtilis sliding response}

$B$. subtilis motility has been extensively studied, and its transcriptional and post-transcriptional regulation have been described in detail $^{23,25,32}$. The master regulator DegU, known to control the production of extracellular enzymes such as the bacillopeptidase (encoded by the bpr gene) ${ }^{33}$, also coordinates motility ${ }^{34}$, colony architecture $^{35}$ and cell differentiation ${ }^{36}$. Biofilm formation, and flagellum and non-flagellum dependent motility are regulated by the transcription factor $\operatorname{Sin}^{37}$. The $B$. subtilis biofilm consists of cells attached to each other by an extracellular matrix, and the production of the matrix occurs in cells with inhibited flagellar motility ${ }^{22}$. The flagellar filament protein that provides the origin to the flagellum is encoded by the hag gene, and the extracellular matrix production is encoded by the epsE-O operon to produce exopolysaccharides (EPS) while the major protein component of the matrix, TasA, is encoded by the yqxM-sipW-tas $A$ operon ${ }^{38}$. Despite the fact that sliding motility does not depend on a flagellum, it does depend on surfactin (encoded by the srfA gene), EPS, and extracellular proteins such as the biofilm-surface layer protein $A(B s \mid A)$ and $\operatorname{Tas}^{25}$.

To identify the genetic requirements for motility, we aimed to test whether a specific process, e.g., motility, matrix production or surfactin production occurs in the $B$. subtilis migratory cells during the interaction assay. To this end, we evaluated the green fluorescent protein (GFP) expression in colonies of our interaction assay. We used $B$. subtilis strains bearing transcriptional fusions of several gene promoters with the gfp gene. Figure $2 a$ shows that both the migratory cells and the $B$. subtilis colony activate the promoters controlling the expression of surfactin (srfA), flagellin (hag), bacillopeptidase (brp) and the BsIA protein (bs/A). Therefore, we investigated their expression at the single-cell level, after dispersing the biofilms, by using flow cytometry (see "Methods" section). Figure $2 \mathrm{~b}$ shows the percentages of cells with $\operatorname{srf} A$, hag, $b r p, b s / A$ expression, compared to the $B$. subtilis wild type (B-WT), as single colonies (left) and in the interaction assays against S. epidermidis (right), at three sampling points $(24,48$, and $72 \mathrm{~h}$ ). The single-cell measurements allowed us to identify two highly activated subpopulations: motile $B$. subtilis cells (hag) and surfactin-secreting (srfA) cells (Fig. S3). This result indicates that these $B$. subtilis subpopulations underline the bacterial interaction against $S$. epidermidis. For instance, we observe that at an early incubation time $(24 \mathrm{~h})$, hag is highly expressed $(65 \%$ in the interaction assay), whereas at late incubation time (72 h), surfactin (srfA, highly expressed in $91 \%$ cells of the interaction assay) plays an important role. Our data is in agreement with the observation of complete engulfment of the S. epidermidis colony after $72 \mathrm{~h}$ incubation in our interaction assays.

Next, we tested the migratory response of $B$. subtilis deletion mutants of key genes involved in motility, matrix production and motility. Figure $2 b$ shows the effect of strains affected in the production of the extracellular matrix (TasA, EPS) on motility and antimicrobial production. A slight defect in migratory response for the eps $G$ mutant, and no motility defects are observed for the tas $A$ mutant. With respect to surfactin, the deletion of srfA results in a low motility response. Remarkably, the hag mutant shows a reduced migratory response (Fig. 2C and Fig. S4), and since sliding represents a flagellum-independent motility, this result confirms that $B$. subtilis employs sliding motility in our interaction assays. We also evaluated the deletion mutants of the main regulators DegU and SinR. Importantly, the lack of DegU results in a lack of antimicrobial production and absence of the migratory response (Fig. 2c and S5). In contrast, the lack of SinR results in antimicrobial 
a

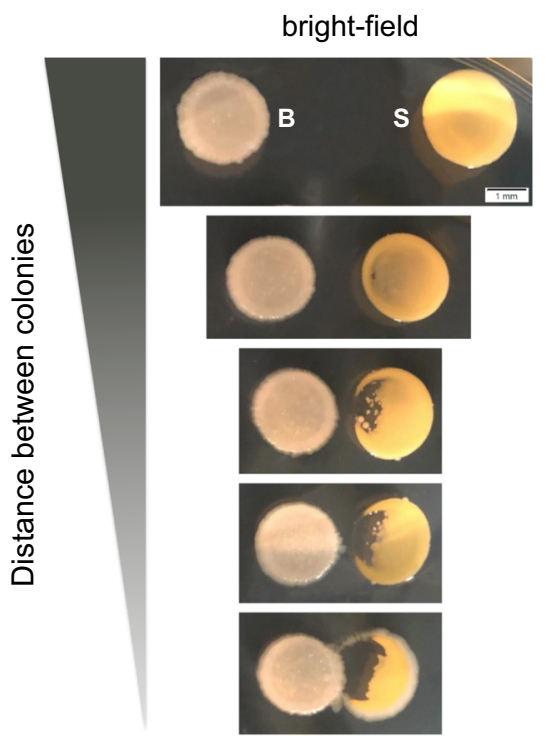

C

$24 \mathrm{~h}$

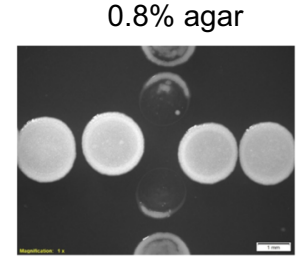

$48 \mathrm{~h}$

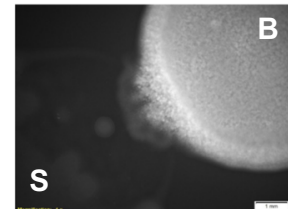

B

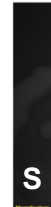

$1 \%$ agar

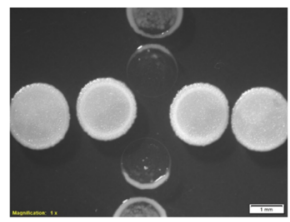

B

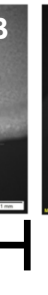

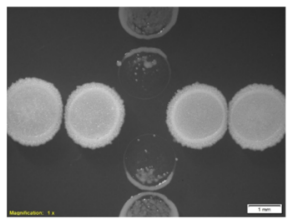

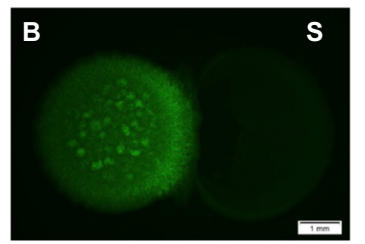

$48 \mathrm{~h}$

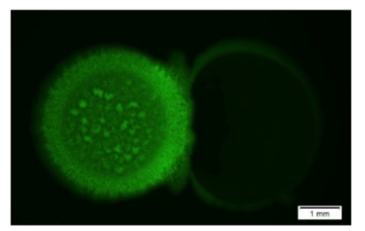

$76 \mathrm{~h}$
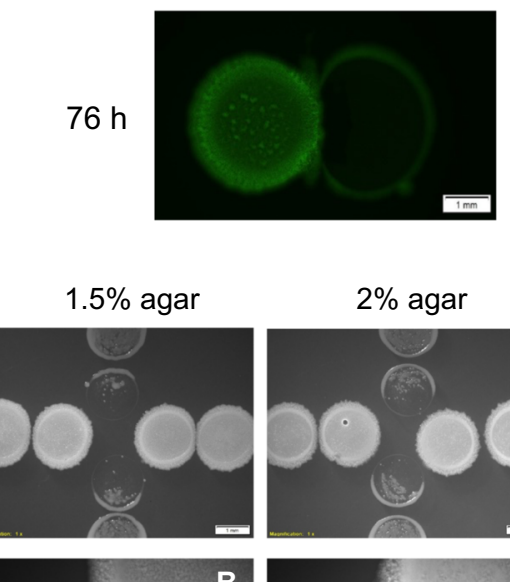

B

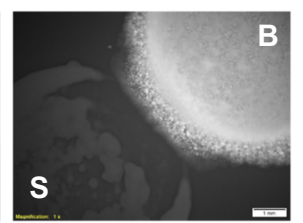

S

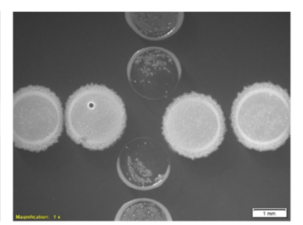

swimming, swarming

Fig. 1 B. subtilis antimicrobial production and sliding in the proximity of S. epidermidis. a Interaction assay of bacterial colonies of $B$. subtilis (B) and S. epidermidis (S) at different separation distances on CDM-agar plates, after $28 \mathrm{~h}$ incubation at $37^{\circ} \mathrm{C}$. Snapshots of microscope observations bright field are shown. Scale bar, $1 \mathrm{~mm}$. b Interaction between bacterial colonies at different incubation times. A B. subtilis GFP+ (B) and S. epidermidis GFP- (S) are shown. Snapshots of observations by fluorescence microscopy are shown. Scale bar, $1 \mathrm{~mm}$. C, B. subtilis motility in CDM-agar plates with varying concentrations of agar (0.8, 1, 1.5, and 2\%). Observations after $24 \mathrm{~h}$ incubation (top images) of a T-shape interaction assay where $B$. subtilis (colonies located at the horizontal line) and S. epidermidis (colonies located at the vertical line) are shown. Observations after $48 \mathrm{~h}$ incubation highlight the B. subtilis (B) motility towards $\mathrm{S}$. epidermidis (S). Snapshots of observations by fluorescence microscopy are shown. Scale bar, $1 \mathrm{~mm}$.

production, but no migratory response. Previous studies conclude that $\operatorname{Sin} R$ is a regulator that controls the transition between motile and non-motile cells in order to form multicellular communities ${ }^{39}$.

As noted in Fig. 2a, the production of the extracellular enzyme bacillopeptidase $(b p r)$ does not play a role in this bacterial interaction. We further studied the role of extracellular enzymes by using B. subtilis WB800, an eight protease-deficient strain (nprE, nprB, aprE, epr, bpr, mpr, vpr, wprA $)^{40}$. Fig. S6 shows that $B$. subtilis WB800 is able to degrade the $S$. epidermidis colony. Therefore, we discarded the possibility that the extracellular proteases are responsible for the $S$. epidermidis colony degradation.

Together, these findings show that $B$. subtilis develops sliding motility, involving motile cells ( $h a g$ ) and surfactin-expressing cells (srfA), to reach and surround $S$. epidermidis. The regulator SinR coordinates this process. In addition, the master regulator DegU not only participates in controlling the motility response, but also in the antimicrobial production against S. epidermidis.
Development of antimicrobial resistance in S. epidermidis

Next, we performed an interaction assay where $B$. subtilis and $S$. epidermidis colonies have a separation distance that does not allow $B$. subtilis to slide and reach the neighbor colony, but it does allow the antimicrobial compound produced by $B$. subtilis to diffuse and degrade (part of) the neighbor colony. Resistant cells of $S$. epidermidis emerge and give rise to bacterial colonies after six days of incubation. Figure 3a shows several resistant colonies, and four of them (indicated with numbers) were selected to gain insight into the nature of the antimicrobial compound secreted by $B$. subtilis. Next, we confirmed the resistance of the selected $S$. epidermidis strains in an interaction assay against $B$. subtilis. Figure $3 \mathrm{~b}$ shows that the highest antimicrobial resistance is observed in the strains 1 (S-1) and 3 (S-3), whereas the strains 2 (S-2) and 4 (S-4) are partially resistant compared to the wild type (S-WT). To investigate whether genetic mutations are responsible of the resistant phenotype, the genomes of S-1 and S-3 were sequenced. The genomic sequence data reveals that these strains 
a
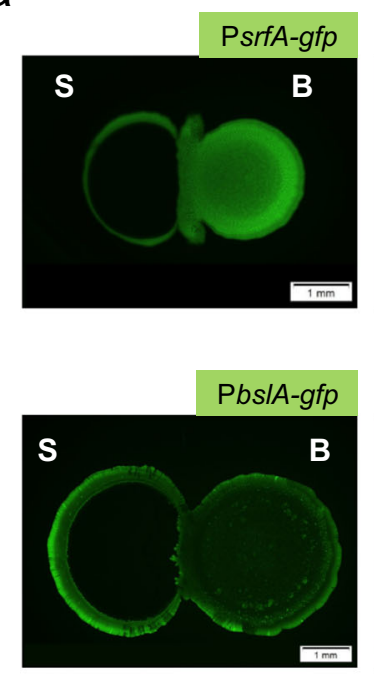
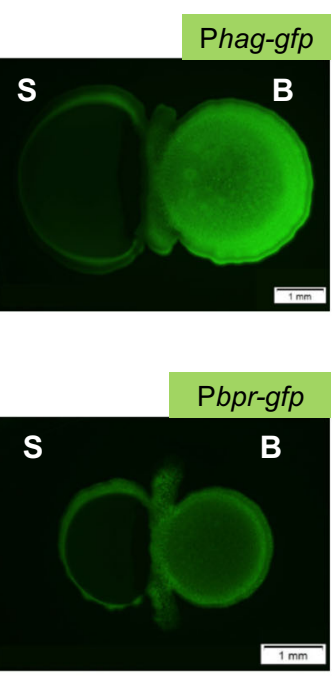

C
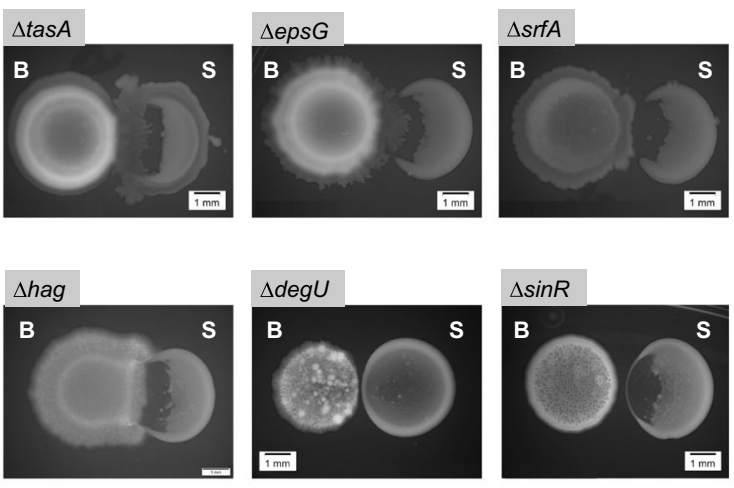

$\Delta \sin R$
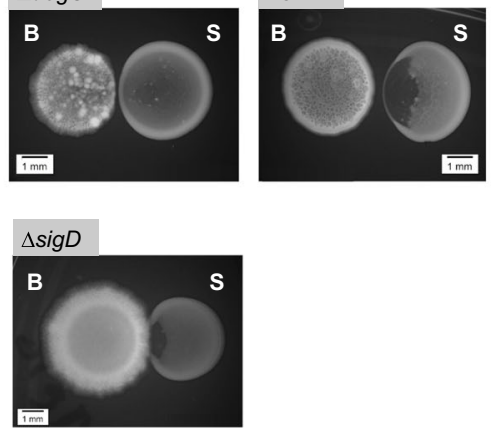

b

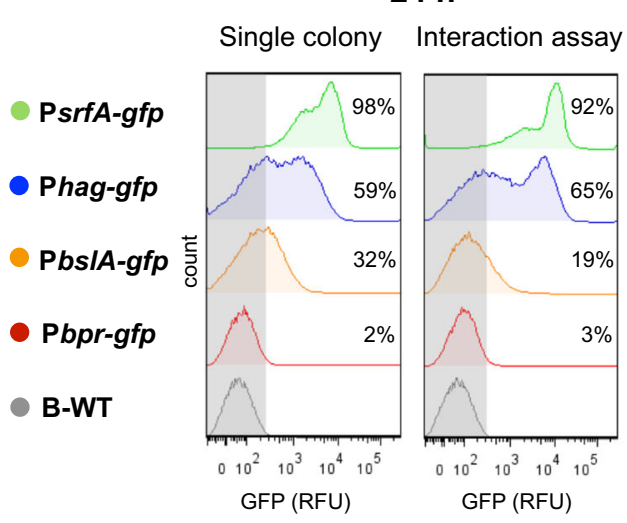

$48 \mathrm{~h}$

Single colony Interaction assay
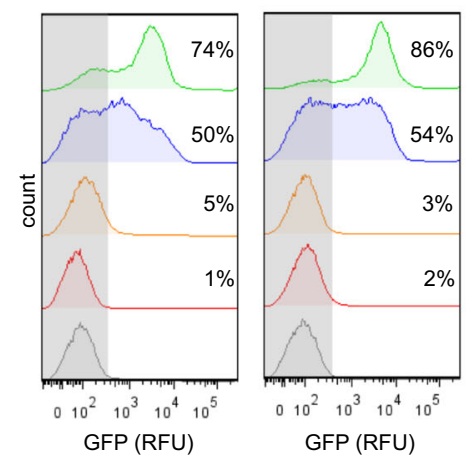

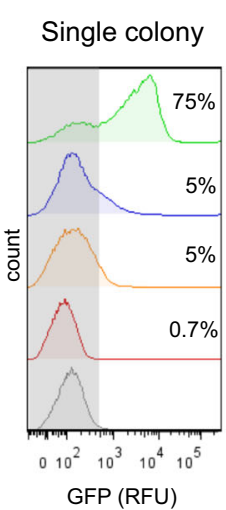

$72 \mathrm{~h}$

nteraction assay

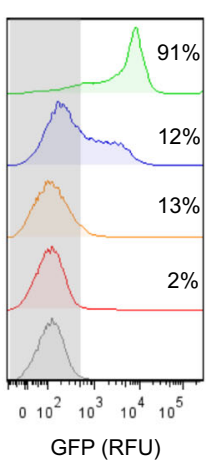

Fig. 2 Genetic elements of $\boldsymbol{B}$. subtilis involved on its antimicrobial and motility response. a $B$. subtilis (B) strains bearing transcription fusions of promoters (PsrfA, Phag, PbslA, Pbpr) with the gene encoding the green fluorescent protein (gfp) in interaction assays against $S$. epidermidis (S). Snapshots of colonies on CDM-agar plates incubated at $37^{\circ} \mathrm{C}$ for $72 \mathrm{~h}$. Scale bar, $1 \mathrm{~mm}$. b Single-cell fluorescence measurements by flow cytometry, in the B. subtilis (B) strains bearing transcription fusions of promoters (PsrfA, Phag, PbslA, Pbpr) with the gene encoding the green fluorescent protein $(g f p)$ in single colonies (left) and interaction assays against $S$. epidermidis ( $S$; right), at different incubation times $(24,48$, and $72 \mathrm{~h}$ ). The percentage of cells with higher gene expression above the background fluorescence level (above the B-WT fluorescence; indicated with a gray window) is shown. 30,000 ungated events for each sample are shown. c $B$. subtilis deletion mutants (tasA, epsG, srfA, hag, $\operatorname{deg} U$, and $\sin R$ ) in in interaction assays against $S$. epidermidis (S). Snapshots of colonies on CDM-agar plates incubated at $37^{\circ} \mathrm{C}$ for $48 \mathrm{~h}$. Scale bar, $1 \mathrm{~mm}$.

have mutations located in only two coding genes, i.e., a point nonsense mutation in the $d t p T$ gene encoding the dipeptide transport system DtpT and several mutations in the bap gene encoding the biofilm-associated protein Bap. Remarkably, the mutation in $d t p T$ causes a non functional dipeptide transport system. Therefore, this finding suggests that a dipeptide with antimicrobial activity is produced by $B$. subtilis against $S$. epidermidis.

\section{Chlorotetain production and genetic regulation}

Previous studies on non-ribosomal peptides (NRPs) produced by $B$. subtilis have shown that this bacterium is able to secrete bacilysin (also known as tetaine) and its chlorinated derivatives bromotetain and chlorotetain ${ }^{14}$. Bacilysin is the simplest antibiotic known, made up of $\mathrm{L}$-alanine and $\mathrm{L}$-anticapsin ${ }^{30}$. This dipeptide is active against bacteria and some fungi like Candida albicans ${ }^{41}$. It is transported into the host cells, and its hydrolyzation by peptidases releases the L-anticapsin, which inhibits the glucosamine 6-phosphate (GlcN6P) synthase, resulting in cell death $^{30,42}$. Interestingly, DegU was shown to positively regulate the biosynthesis of bacilysin in Bacillus amyloliquefaciens ${ }^{43}$. DegU and the DegS kinase are a two component signal system that controls several cellular processes, including motility and biofilm formation ${ }^{44}$. Thus, we tested a degU deletion mutant in the interaction assay against $S$. epidermidis. Figure $4 a$ shows that the lack of DegU results in very low levels of $S$. epidermidis colony degradation, compared to the interaction with wild-type $B$. subtilis. Moreover, overnight $B$. subtilis bacterial supernatants of both wild type and $\Delta d e g U$ strains were tested for antimicrobial production using S. epidermidis as indicator strain (Fig. 4b). Accordingly, the supernatant of the $\Delta d e g U$ strain shows no antimicrobial activity. We performed ultra high-performance liquid chromatography coupled with mass spectrometry (UHPLC-M) to identify the chemical structure of the antimicrobial compound. The differential analysis of the compounds present in both bacterial supernatants (wild type and $\Delta$ degU; Fig. 4c, d, respectively) clearly shows that 
a
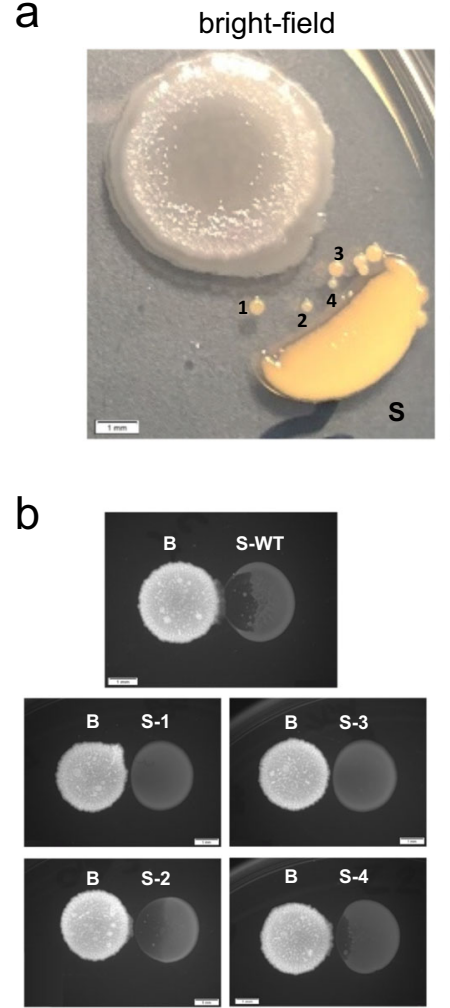

GFP

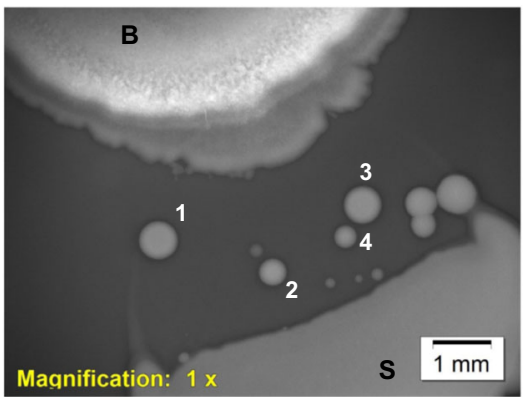

C

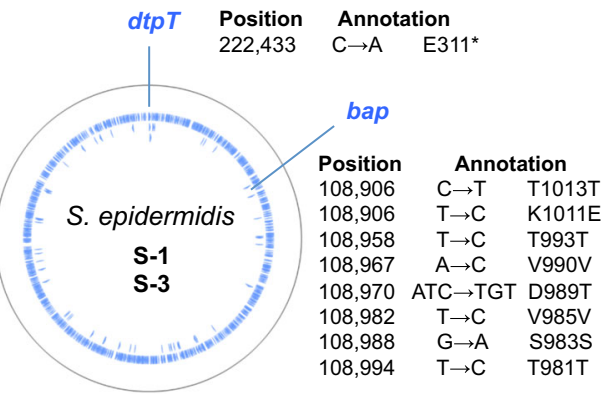

Fig. 3 S. epidermidis resistant colonies emerge. a Interaction assay of B. subtilis (B) and S. epidermidis (S) colonies on CDM-agar plates incubated at $37^{\circ} \mathrm{C}$ for 6 days. Resistant colonies selected are indicated with numbers ( 1 to 4). Snapshots of observations with bright-field (left) and fluorescence (right) microscopy are shown. Scale bar, $1 \mathrm{~mm}$. b Interaction assay with B. subtilis wild type strain (B) and S. epidermidis wild type (S-WT) and selected mutants (S-1, S-2, S-3, and S-4). Snapshots of observations with fluorescence microscopy are shown. Scale bar, 1 mm. c Mutations in the genomic sequence of both S. epidermidis S-1 and S-3 strains. Location and description of mutations in the dtpT and bap genes are indicated.

the wild-type strain secretes the bacilysin derivative, chlorotetain (Fig. 4c). This result is in contrast to the supernatant of the $\Delta$ degU strain, which does not contain chlorotetain (Fig. 4d). Chlorotetain is detected at $0.8273 \mathrm{~min}$ with $\mathrm{m} / \mathrm{z}=289.09\left([\mathrm{M}+\mathrm{H}]^{+}\right)$.

The enzymes participating in the synthesis of bacilysin are encoded in the bacABCDE (ywfBCDEF) gene cluster of $B$. subtilis ${ }^{45}$. We aimed to confirm that bacilysin is solely responsible for the S. epidermidis colony degradation. To this end, we performed an interaction assay with two bacilysin mutant strains $(B$. subtilis $\triangle b a c A$ and $\triangle b a c D$ ). Figure 5 a shows that the bacilysin mutants are unable to degrade the $S$. epidermidis colony. In addition, the bacterial supernatant of any of bacilysin mutants shows no antimicrobial activity compared to the growth inhibition by the supernatant of the wild-type B. subtilis (B-WT) strain (Fig. 5b). Altogether, these results confirm that chlorotetain is solely responsible for the $S$. epidermidis colony degradation.

The gene regulation by DegU is dependent on its phosphorylation (DegU-P) by the DegS kinase ${ }^{36}$. Both forms of DegU, phosphorylated and non-phosphorylated, control different cellular processes. For instance the extracellular production of degradative enzymes requires high levels of DegU- $\mathrm{P}^{44}$. A previous study on bacilysin biosynthesis by $B$. amyloliquefaciens shows that both DegU and DegU-P are able to bind the bacABCDE operon promoter to produce bacilysin ${ }^{43}$. Based on this evidence, we next analyzed the contribution of DegU and DegU-P to the production of bacilysin in vivo. To achieve this aim, we used two $B$. subtilis strains with different phosphorylation levels of DegU. The degU32 mutant has been reported to show high levels of the phosphorylated form of DegU (DegU-P), whereas the degU146 mutant shows low levels of DegU-P ${ }^{46}$. We used these degU mutant strains in the interaction assay. Figure $5 c$ shows increased bacilysin production by the $B$. subtilis degU32 mutant and reduced bacilysin production by the degU146 mutant. In addition, we identified the presence and concentration levels of chlorotetain in bacterial supernatants of the degU mutants by UHPLC-M. Figure $5 \mathrm{~d}$ shows that the degU32 strain produces chlorotetain at higher concentrations than the wild type (B-WT), whereas chlorotetain is not detected in the bacterial supernatant of the deg146 strain. Altogether, we propose a model where DegU-P promotes bacilysin production by $B$. subtilis, and higher or lower levels of DegU phosphorylation result in increased or reduced bacilysin production, respectively.

\section{Cell-to-cell attachment indirectly triggers motility}

Regarding the factors involved in the $S$. epidermidis triggered $B$. subtilis motility, we investigated whether the intracellular or extracellular content of S. epidermidis cells promote the B. subtilis motility. To this end, we evaluated the intracellular and extracellular content of $S$. epidermidis cells in a disc assay (Fig. S7) and observed that the extracellular content triggers the B. subtilis motility.

As mentioned above, S. epidermidis S-1 and S-3 are not only resistant to chlorotetain, but also do not promote motility by $B$. subtilis. Therefore, we tested whether overnight $S$. epidermidis supernatants (S-WT, S-1, and S-3) are able to promote motility by $B$. subtilis. To this end, $B$. subtilis cells were spotted in a spiral configuration to show that colonies in the proximity to the supernatant are able to show the typical migratory response. Figure $6 a$ shows that $B$. subtilis colonies (indicated with a B letter) exclusively develop motile cells towards the $S$. epidermidis S-WT supernatant located in the center of the agar plate (indicated with 
a
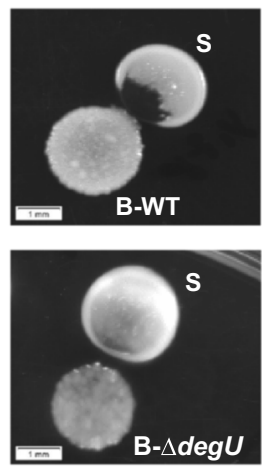

C
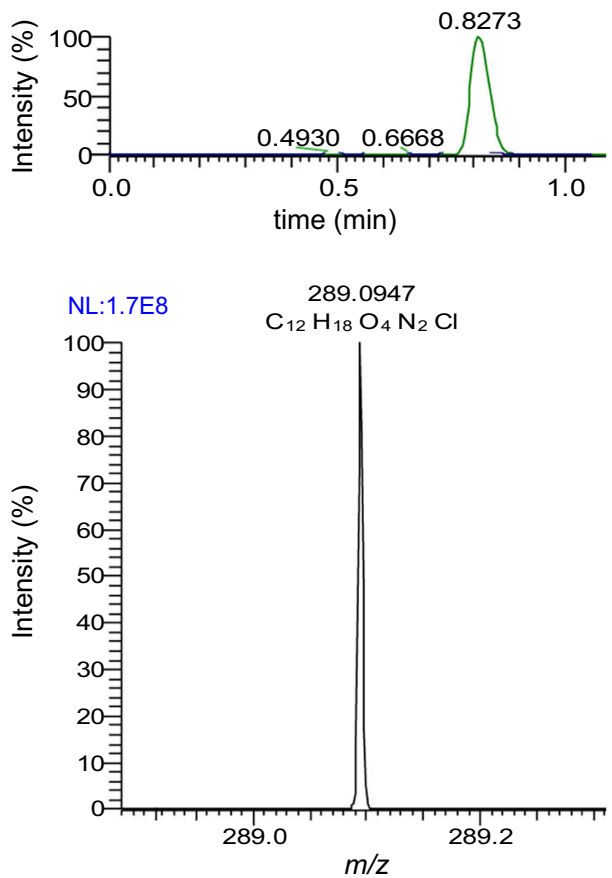

e

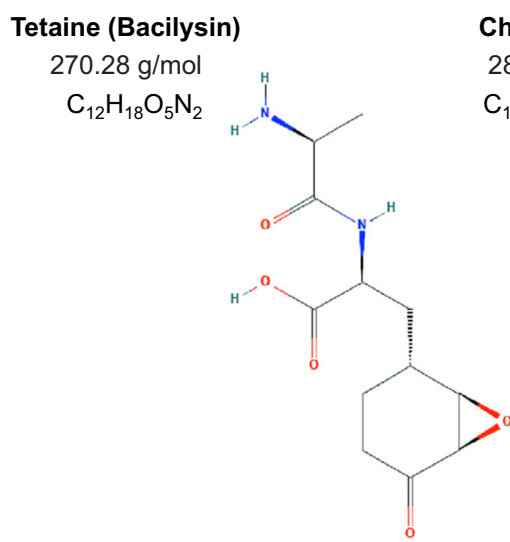

b

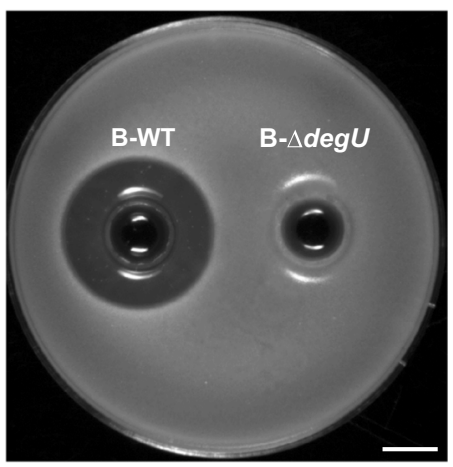

d
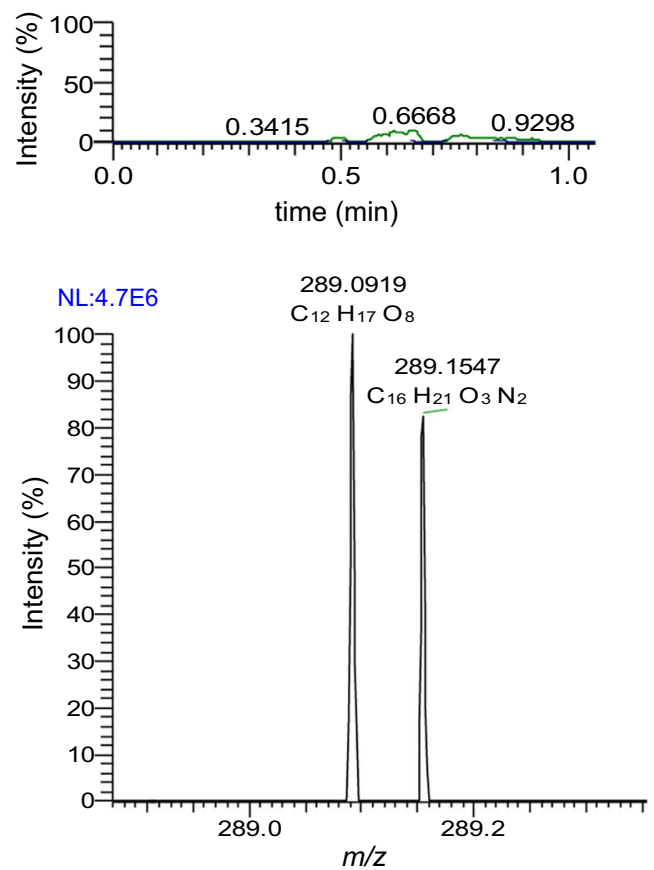

Chlorotetaine

$288.73 \mathrm{~g} / \mathrm{mol}$

$\mathrm{C}_{12} \mathrm{H}_{17} \mathrm{O}_{4} \mathrm{~N}_{2} \mathrm{Cl}$

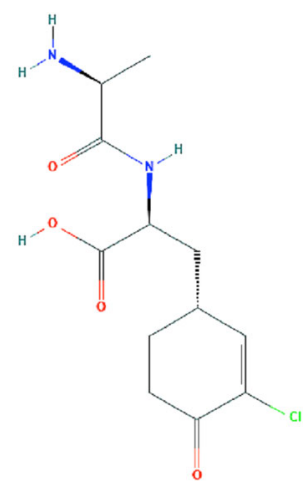

the letter S), compared to the control (CDM indicated with the letter C) and the S-1 or S-3 supernatant. A very low level of $B$. subtilis motility, at the closest distance to the supernatant, is slightly observed towards the S-1 supernatant. Figure $3 \mathrm{c}$ shows that the mutations in the biofilm-associated protein are located in a narrow range of residues of the protein sequence (981-1013). Thus, we analyze the Bap protein sequence in order to identify protein domains and gain an insight into the consequences of the mutations on the phenotype of the S-1 and S-3 strains. The sequence analysis reveals that the mutations target a cadherin repeat-like domain (Fig. 6b). Bap proteins are involved in attachment of cells to surfaces and intercellular adhesion. Previous studies show that Bap can sense the bacterial environment through $\mathrm{Ca}^{2+}$ concentration changes and trigger the cell-to-cell 
Fig. 4 Chlorotetain production by B. subtilis. a Interaction assay between B. subtilis wild type (B-WT) and S. epidermidis wild type (S) strains (top image), and between $B$. subtilis degU deletion mutant (B- $\Delta \operatorname{deg} U$ ) and $S$. epidermidis wild type (S) strains (bottom image). Snapshots of colonies on CDM-agar plates incubated at $37^{\circ} \mathrm{C}$ for $24 \mathrm{~h}$. Scale bar, $1 \mathrm{~mm}$. b Antimicrobial activity of overnight bacterial supernatants of $B$. subtilis wild type (B-WT; left) and B. subtilis degU deletion mutant (B- $\Delta$ degU) strains on CDM-agar plates. S. epidermidis wild type strain was used as indicator strain. A snapshot of the antimicrobial activity, indicated by the $S$. epidermidis growth inhibition halo, was taken after incubation at $37^{\circ} \mathrm{C}$ for $24 \mathrm{~h}$. Scale bar, $15 \mathrm{~mm}$. c, d UHPLC chromatograms (top, $x$-axis indicates time in minutes, and $y$-axis indicates percentage of signal intensity) and mass spectra (bottom, $x$-axis indicates $\mathrm{m} / \mathrm{z}$, mass-to-charge ratio; and $y$-axis indicates percentage of signal intensity), respectively, to confirm the presence of chlorotetain in overnight bacterial supernatants of $B$. subtilis wild type (c) and $B$. subtilis deg $U$ deletion mutant $\mathbf{d}$. The molecular weight of the peak of the mass spectrum in c corresponds to chlorotetain. e, chemical structures of bacilysin (left) and chlorotetain (right), the molecular weight and chemical formula is indicated.

attachment or biofilm formation ${ }^{47}$. Indeed, cadherin repeat-like motifs are potential $\mathrm{Ca}^{2+}$ binding motifs ${ }^{48}$.

Since Bap proteins are responsible for cell-to-cell attachment, and potentially contribute to biofilm formation, we evaluated the attachment of cells of S. epidermidis wild type and S-3 strains to polystyrene plates in CDM supplemented with $\mathrm{Ca}^{2+}$. We performed a biofilm assay based on crystal violate (CV) in a qualitative (Fig. 6c) and quantitative (Fig. 6d) way, and used B. subtilis to compare the biofilm potential of the S. epidermidis strains. Figure $6 \mathrm{c}$, d show that cells of S. epidermidis S-3 are affected by the presence of $\mathrm{Ca}^{2+}$, compared to the wild-type cells (S-WT), which show the same attachment properties when $\mathrm{Ca}^{2+}$ is present. Interestingly, the $B$. subtilis biofilm is strongly affected by the presence of $\mathrm{Ca}^{2+}$. The effects of $\mathrm{Ca}^{2+}$ on colony biofilms of $B$. subtilis have been previously studied, colony expansion is observed at low concentrations of calcium ions and a mechanism where $\mathrm{Ca}^{2+}$ modifies the amphiphilic properties of surfactin is proposed $^{49}$.

Furthermore, in a preliminary study between several $B$. subtilis and $S$. epidermidis strains, we observed that $B$. subtilis does not develop motility towards a $S$. epidermidis Bap negative strain (Fig. S8). To further confirm this observation, we collected a bacterial supernatant of the $S$. epidermidis Bap negative strain and tested the $B$. subtilis motility as described above. Fig. S9 shows that only the S. epidermidis S-WT supernatant (Bap positive) triggers the $B$. subtilis motility. This result further supports a model where the Bap proteins are responsible of promoting $B$. subtilis motility.

Since EDTA is a calcium-chelating agent, we attempted to evaluate the interaction between $B$. subtilis and $S$. epidermidis in CDM-agar plates supplemented with EDTA. To this end, we used CDM containing 2.5 and $0.25 \mathrm{mM}$ EDTA. Fig. S10 shows that EDTA strongly affects the $B$. subtilis growth. $B$. subtilis is unable to grow in the presence of EDTA $2.5 \mathrm{mM}$, and even at lower EDTA concentration $(0.25 \mathrm{mM})$, where it is able to grow, it is unable to produce chlorotetain and motility. In addition, Fig. S10b shows that the S. epidermidis WT colony expansion is higher than the S-3 mutant in the presence of EDTA $0.25 \mathrm{mM}$. Since the S-3 strain has several mutations in the calcium-binding domain of the Bap protein, we speculate that the phenotype of lower colony expansion is related to the Bap protein and its role in cell-to-cell attachment. A deep biochemical analysis of the Bap protein in the S-1 and S-3 strains to investigate the molecular mechanism involved in the calcium binding or sensing should be undertaken to further corroborate the mechanism.

Together, our data suggest that the mutations in the Bap protein of S. epidermidis (S-1 and S-3 strains) potentially affect the cell-to-cell attachment and cell aggregation via $\mathrm{Ca}^{2+}$ sensing (see Fig. S4). Since $S$. epidermidis mutants are unable to promote motility in $B$. subtilis, and low concentrations of $\mathrm{Ca}^{2+}$ trigger colony expansion in $B$. subtilis ${ }^{49}$, we propose that the $B$. subtilis sliding occurs in response to the low availability of $\mathrm{Ca}^{2+}$ ions, which are sequestered by Bap proteins in the proximity of the S. epidermidis colony.

\section{DISCUSSION}

Bacteria commonly live in densely populated environments, where competition for nutrients and space is crucial ${ }^{10}$. The skin is one of these dense environments, where bacterial interactions occur to maintain a balance, and thus determine the composition of our skin microbiota ${ }^{50}$. We use $B$. subtilis and $S$. epidermidis to study whether these bacteria are able to establish a type of specific bacterial interaction. $B$. subtilis is recognized as an important protective bacterium of our $\operatorname{skin}^{16}$ and $S$. epidermidis is the most abundant member of the skin microbiota ${ }^{51}$. Our study reveals an interaction between these bacteria based on chlorotetain production and development of $B$. subtilis motile cells. Figure 7 shows a proposed model of this interaction, where the first response from B. subtilis colonies ( $24 \mathrm{~h}$ of incubation) is characterized by chlorotetain production, which results in partial degradation of the $S$. epidermidis colony. Previous studies suggest that bacilysin has other cellular functions beyond its antimicrobial activity, for instance bacilysin negative strains are defective in sporulation or germination/outgrowth ${ }^{52}$. In addition, bacilysin is highly produced during starvation stress ${ }^{30}$. Thus, $B$. subtilis might benefit of bacilysin (or chlorotetain) production, when growing in poor-nutrient niches, in two ways: regulation of cellular processes and killing competitors. These observations might explain why to utilize a very simple (dipeptide) antimicrobial instead of others to conquer a nutrient-limited environment. The second response involves an emerging $B$. subtilis subpopulation with sliding motility. Previous studies have demonstrated the capacity of $B$. subtilis to engulf other bacterial species ${ }^{31,53,54}$. We propose that in our study, this motility occurs as a response to the low $\mathrm{Ca}^{2+}$ levels in the environment due to the $\mathrm{Ca}^{2+}$ binding to the biofilmassociated protein Bap within the $S$. epidermidis colony and its surroundings. We propose that this spreading property by $B$. subtilis is a niche conquering strategy to expand its bacterial colony and limit the growth of other species.

Bacterial interactions are shaped by several factors, for instance by the cell density and the proximity between cells ${ }^{55}$. In our study, the interactions between $B$. subtilis and $S$. epidermidis colonies are indeed affected by the initial cell density. For instance, we observed that the lower the $S$. epidermidis initial cell densities, the higher the colony degradation (Fig. S11). This effect can be explained by the fact that at low $S$. epidermidis cell densities, chlorotetain is quickly produced by $B$. subtilis (present at higher cell densities), then it accumulates in the growth medium and immediately kills the low number of $S$. epidermidis cells.

The avoidance of conflicts and fights is a common strategy to survive between animals ${ }^{56-58}$. Here, we uncover an interaction between two microorganisms that resembles examples of the conflicts observed between higher organisms, for instance when an animal backs down if faced with another animal in competition. In this case study, our data suggest that $S$. epidermidis is actually playing a role in the interaction with $B$. subtilis by limiting the incoming attacks, e.g., it develops resistance to chlorotetain and mutations in the bap gene arises, which results in abolishment of the $B$. subtilis invasion of its colony. This strategy results in an effective way to allow S. epidermidis to survive. We 
a
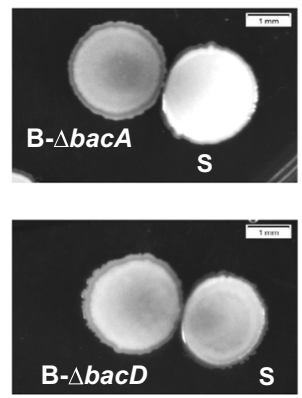

C
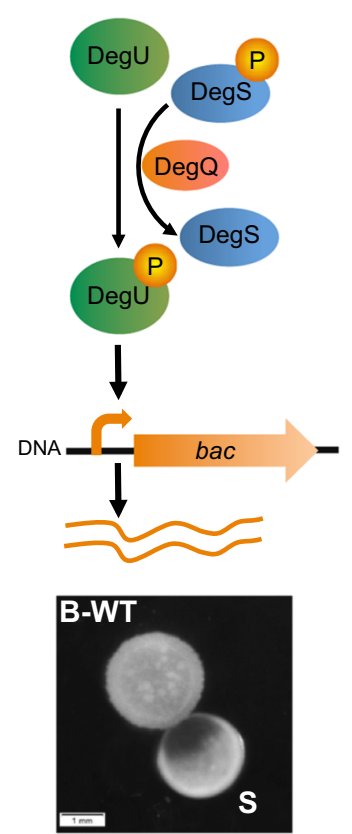

b
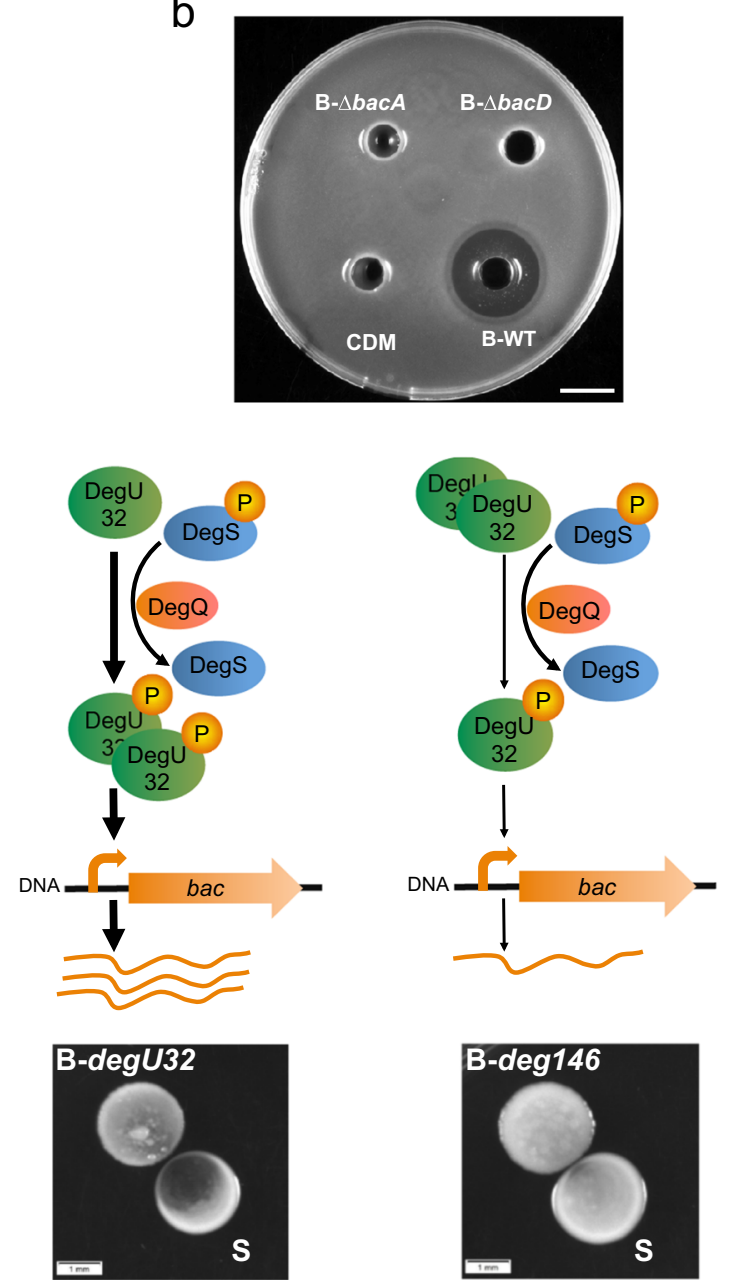

d
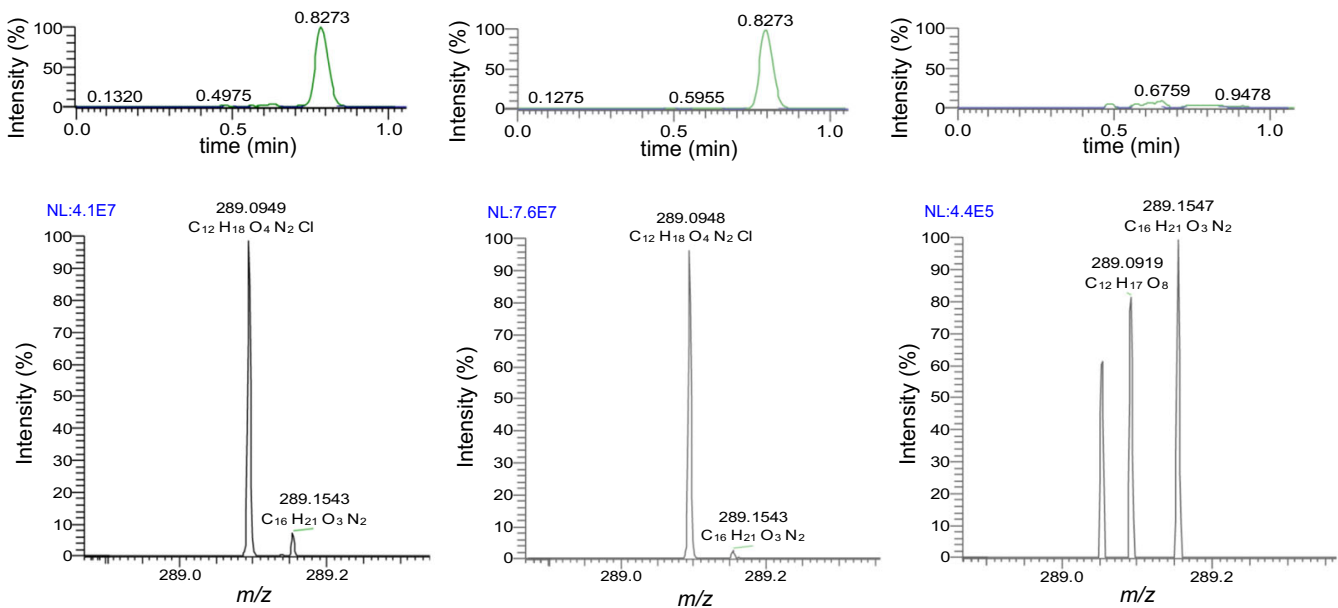

observed that S. epidermidis resistant colonies show lower growth rate and reach lower cell densities compared to the wild type (Fig. S12). Thus, the resulting genetic mutations on the $d t p T$ and bap genes lower the $S$. epidermidis fitness probably because of their impact on essential processes such as the nutrient uptake (via DtpT), and cell-to-cell adhesion (via Bap). Furthermore, as mentioned above, cell density also plays an important role in this interaction. Thus, the unaffected S. epidermidis cells (initially at a far distance from the $B$. subtilis colony), might not develop chlorotetain resistance, but are able to quickly grow and reach a high cell density, becoming a bacterial population able to tolerate high chlorotetain concentrations.

This study sheds light on how microbial communities define their territory in certain microenvironments of our skin, where many other bacteria live. Notably, $B$. subtilis is able to produce a plethora of antimicrobial compounds, which show activity against 
Fig. 5 Genetic elements involved in chlorotetain production by B. subtilis. a Interaction assay between bacilysin $B$. subtilis mutants (B- $\Delta b a c A$ and $\mathrm{B}-\Delta b a c D$ ) with the wild-type $S$. epidermidis (S) strain, top and bottom images, respectively. Snapshots of colonies on CDM-agar plates incubated at $37^{\circ} \mathrm{C}$ for $24 \mathrm{~h}$. Scale bar, $1 \mathrm{~mm}$. b Antimicrobial activity of overnight bacterial supernatants of the bacilysin $B$. subtilis mutant (B- $\triangle b a c A$ and $\mathrm{B}-\Delta b a c D)$ and wild-type $B$. subtilis (B-WT) strains on CDM-agar plates. S. epidermidis wild-type strain was used as indicator strain. $\mathrm{CDM}$, used for bacterial growth, is shown as a negative control. Snapshot of the antimicrobial activity, indicated by the $S$. epidermidis growth inhibition halo, was taken after incubation at $37^{\circ} \mathrm{C}$ for $24 \mathrm{~h}$. Scale bar, $10 \mathrm{~mm}$. c Proposed model (top) of the relationship between the phosphorylation of DegU (highlighted with different thickness in the arrow), and its effect on bacilysin biosynthesis. Corresponding interaction assays (bottom) between B. subtilis degU mutants (B-degU32 and B-degU146; center and right, respectively) strains targeting the DegU phosphorylation levels and S. epidermidis wild type (S) strain. An interaction assay between wild-type strains of $B$. subtilis (B-WT) and S. epidermidis (S) was used as a control (left). Snapshots of colonies on CDM-agar plates incubated at $37^{\circ} \mathrm{C}$ for $24 \mathrm{~h}$. Scale bar, $1 \mathrm{~mm}$. d, UHPLC chromatograms (top, $x$-axis indicates time in minutes, and $y$-axis indicates percentage of signal intensity) and mass spectra (bottom, $x$-axis indicates $\mathrm{m} / \mathrm{z}$, mass-to-charge ratio; and $y$-axis indicates percentage of signal intensity), respectively, to confirm the concentration levels of chlorotetain in overnight bacterial supernatants of $B$. subtilis degU mutants (B-degU32 and B-degU146; center and right, respectively) compared to the $B$. subtilis wild type (left). The molecular weight $\left(289.09 \mathrm{~g} \mathrm{~mol}^{-1}\right)$ of the peaks of the mass spectra corresponds to chlorotetain.

diverse bacteria and fungi ${ }^{14,59}$. For instance $B$. subtilis produces bacillomycin, an antibiotic with antifungal activity against all the important dermatophytes and systemic fungi ${ }^{60}$. Likewise S. epidermidis is able to produce peptides to inhibit Staphylococcus aureus and Streptococcus pyogenes ${ }^{2}$.

The skin microbiota is composed of many other different species of bacteria ${ }^{2,3}$. Thus, it is interesting to speculate about different interaction scenarios between these bacteria. Our study shows how bacteria respond locally to the secreted products of others, for instance the $S$. epidermidis resistance response when inhibitory molecules are in the environment. In addition, species of bacteria are known to colonize specific microenvironments of our skin, based on different factors such as $\mathrm{pH}$, nutrients, or humidity ${ }^{61}$. Accordingly, specific bacterial competition strategies appear to be an important factor that defines which bacteria colonize a specific microenvironment of our skin.

\section{METHODS}

Bacterial strains and growth conditions

All the bacterial strains used for this study are listed in the Supplementary Table 1. Experiments were performed with Bacillus subtilis 168, and Staphylococcus epidermidis $\mathrm{JH}$ (wild type). Staphylococcus epidermidis $\mathrm{JH}$ was isolated from human skin in Groningen, The Netherlands, by using an inoculation loop, passing it onto the surface of a human arm, and then streaking an M17-agar plate. A colony with beige color was obtained after $24 \mathrm{~h}$ incubation at $37^{\circ} \mathrm{C}$. The colony was again streaked out on an M17agar plate to ensure the isolation of a single bacterium. Initially, the sequence analysis of 16S rRNA gene was performed using the BLAST program to identify this bacterium as Staphylococcus epidermidis ${ }^{62}$. Confirmation of the taxonomic assignment was performed by genomic DNA sequencing (see Genome sequencing).

B. subtilis, S. epidermidis, C. acnes, S. thermophilus cells were grown at $37^{\circ} \mathrm{C}$ in chemically defined medium $(\mathrm{CDM})^{63}$, supplemented with glucose (Sigma-Aldrich) $0.5 \%(\mathrm{w} / \mathrm{v})$. CDM contained $49.6 \mathrm{mM} \mathrm{NaCl}, 20.1 \mathrm{mM}$ $\mathrm{Na}_{2} \mathrm{HPO}_{4}, \quad 20.2 \mathrm{mM} \quad \mathrm{KH}_{2} \mathrm{PO}_{4}, \quad 9.7 \mu \mathrm{M} \quad( \pm)$-a-lipoic acid, $2.10 \mu \mathrm{M}$ Dpantothenic acid, $8.12 \mu \mathrm{M}$ nicotinic acid, $0.41 \mu \mathrm{M}$ biotin, $4.91 \mu \mathrm{M}$ pyridoxal hydrochloride, $4.86 \mu \mathrm{M}$ pyridoxine hydrochloride, $2.96 \mu \mathrm{M}$ thiamine hydrochloride, $0.24 \mu \mathrm{M}\left(\mathrm{NH}_{4}\right)_{6} \mathrm{Mo}_{7} \mathrm{O}_{24}, \mathrm{CaCl}_{2} 20.4 \mu \mathrm{M}, 1.07 \mu \mathrm{M} \mathrm{CoSO}_{4}$, $1.20 \mu \mathrm{M} \mathrm{CuSO}{ }_{4}, 1.04 \mu \mathrm{M} \mathrm{ZnSO}{ }_{4}, 20.12 \mu \mathrm{M} \mathrm{FeCl}, 1.46 \mathrm{mM}$ L-alanine, $1.40 \mathrm{mM}$ L-arginine, $0.61 \mathrm{mM}$ L-asparagine, $1.03 \mathrm{mM}$ L-aspartic acid, $0.35 \mathrm{mM}$ L-cysteine, $0.66 \mathrm{mM}$ L-glutamic acid, $0.66 \mathrm{mM}$ L-glutamine, $0.39 \mathrm{mM}$ glycine, $0.16 \mathrm{mM}$ L-histidine, $0.63 \mathrm{mM}$ L-isoleucine, $0.89 \mathrm{mM}$ L-leucine, $1.02 \mathrm{mM}$ L-lysine, $0.27 \mathrm{mM}$ L-methionine, $0.39 \mathrm{mM}$ L-phenylalanine, $3.58 \mathrm{mM}$ L-proline, $1.64 \mathrm{mM} \mathrm{L}$-serine, $0.57 \mathrm{mM}$ L-threonine, $0.18 \mathrm{mM}$ L-tryptophan, $2.76 \mathrm{mM}$ L-tyrosine and $0.73 \mathrm{mM}$ L-valine. GM17-agar or CDM-agar plates were prepared by adding agar $1.5 \%(\mathrm{w} / \mathrm{v})$ and glucose to M17 or CDM, respectively. When necessary, culture media was supplemented with erythromycin $5 \mu \mathrm{gLL}^{-1}$, chloramphenicol $5 \mu \mathrm{g} \mathrm{mL}^{-1}$, kanamycin $10 \mu \mathrm{g} \mathrm{mL}^{-1}$ or spectinomycin $100 \mu \mathrm{g} \mathrm{mL}^{-1}$.

Lactococcus lactis cells were grown at $30^{\circ} \mathrm{C}$ in $\mathrm{M} 17$ broth (Difco ${ }^{\mathrm{TM}} \mathrm{BD}, \mathrm{NJ}$, USA) or in CDM, supplemented with glucose (Sigma-Aldrich) $0.5 \%(\mathrm{w} / \mathrm{v})$.

For microscopy experiments or plate-reader assays, bacterial strains were grown in CDM with glucose $0.5 \%(\mathrm{w} / \mathrm{v})$ and collected by centrifugation from exponential growth cultures (optical density of 0.4 at
$600 \mathrm{~nm})$ and washed three times with phosphate-buffered saline (PBS) solution (pH 7.2) containing: $15.44 \mu \mathrm{M} \mathrm{KH}_{2} \mathrm{PO}_{4}, 1.55 \mathrm{mM} \mathrm{NaCl}$ and $27.09 \mu \mathrm{M}$ $\mathrm{Na}_{2} \mathrm{HPO}_{4}$.

DNA techniques and oligonucleotides

DNA amplifications by PCR were performed using a PCR mix containing Phusion HF Buffer (Thermo Fisher Scientific Inc., MA, USA), $2.5 \mathrm{mM}$ dNTPs mix, Phusion HF DNA polymerase (Thermo Fisher Scientific Inc., MA, USA), primers $(0.5 \mu \mathrm{M}$ each), and $50 \mathrm{ng}$ of S. epidermidis JH chromosomal DNA as template. Oligonucleotides (Supplementary Table 2) were purchased from Biolegio (Nijmegen, The Netherlands). PCRs were performed in an Eppendorf thermal cycler (Eppendorf, Hamburg, Germany). The $16 \mathrm{~s}$ rRNA was amplified by 35 cycles of denaturation $\left(98^{\circ} \mathrm{C}\right.$ for $\left.30 \mathrm{~s}\right)$, annealing $\left(5^{\circ} \mathrm{C}\right.$ or more, lower than $T_{\mathrm{m}}$ for $\left.30 \mathrm{~s}\right)$, and extension $\left(70^{\circ} \mathrm{C}\right.$ for 1 min per $\left.1 \mathrm{Kbp}\right)$. Amplifications were confirmed by $1 \%(\mathrm{w} / \mathrm{v})$ agarose gel electrophoresis method. PCR products were isolated and cleaned-up with a high pure plasmid isolation kit (Roche Applied Science, Mannheim, Germany), according to the protocol of the manufacturer. DNA sequences were obtained by sequencing of the PCR fragments in the genomic region of interest (Macrogen Europe, Amsterdam, The Netherlands).

\section{Interaction assay}

Bacterial interaction assays were performed on CDM-agar plates. B. subtilis and $S$. epidermidis cells were grown overnight in CDM and washed three times with PBS. The optical density at $600 \mathrm{~nm}$ of each bacterial growth was adjusted to 0.4 . A volume of $5 \mu \mathrm{L}$ of each bacterial suspension was spotted on CDM-agar plates at different distances and incubated at $37^{\circ} \mathrm{C}$.

To test swarming, swimming and sliding motility in B. subtilis, CDM-agar plates were prepared with different agar concentrations: $0.8,1,1.5$ and $2 \%(w / v)$

\section{Microscopy observations}

Interaction between colonies of $B$. subtilis and $S$. epidermidis lactis was detected using an Olympus MVX20 macro zoom fluorescence microscope equipped with a PreciseExcite light-emitting diode (LED) fluorescence illumination $(470 \mathrm{~nm})$, GFP filter set (excitation $460 / 480 \mathrm{~nm}$ and emission $495 / 540 \mathrm{~nm}$ ). Bright-field and fluorescent images were acquired with an Olympus XM10 monochrome camera (Olympus Co., Tokyo, Japan).

\section{DNA sequencing}

A single colony S. epidermidis $\mathrm{JH}$ (wild type strain) growing on a CDM-agar plate was selected and grown as standing culture in $5 \mathrm{~mL}$ of CDM broth, supplemented with $0.5 \%(\mathrm{w} / \mathrm{v})$ glucose, and incubated overnight at $37^{\circ} \mathrm{C}$. Cells from the bacterial culture were collected by centrifugation at $6000 \times \mathrm{g}$ for $3 \mathrm{~min}$ in a Microfuge 16 centrifuge (Beckman Coulter, Woerden, The Netherlands). Genomic DNA was isolated with a GenElute bacterial genome DNA kit (Sigma-Aldrich, Munich, Germany) according to the manufacturer's instructions. The resulting genomic DNA was used as template to perform PCRs. The $16 \mathrm{~S}$ rRNA gene was amplified by colony PCR using the oligonucleotides $27 \mathrm{~F}$ and $1492 \mathrm{R}$ (see Supplementary Table $2)$. The DNA sequences were analyzed with BLAST program ${ }^{62}$.

In addition, the genomic DNA of the S. epidermidis mutant strains (S-1 and S-3) was isolated as described above. To this end, all the staphylococci genomes (wild type, S-1 and S-3) were paired-end sequenced at the Beijing Genomics Institute (BGI, Copenhagen N, Denmark) on a BGISEQ-500 
a
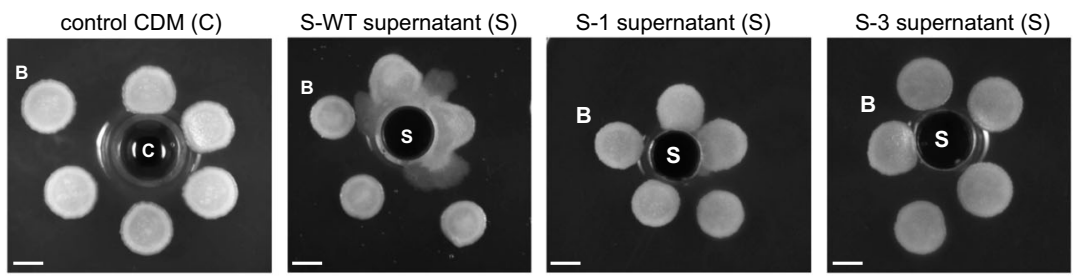

b

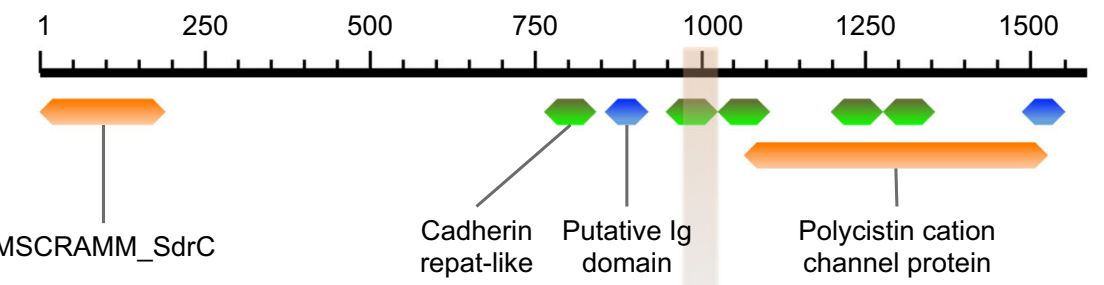

Mutations

In S-1, S-3

C

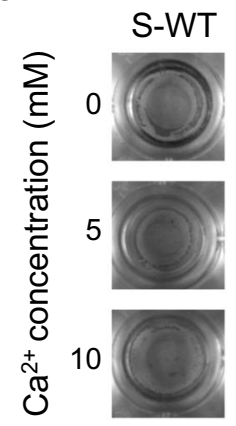

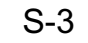

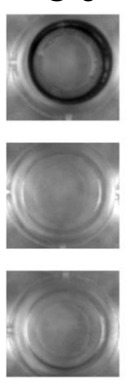

B

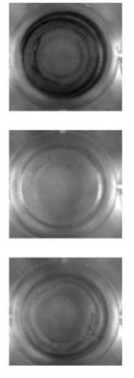

d

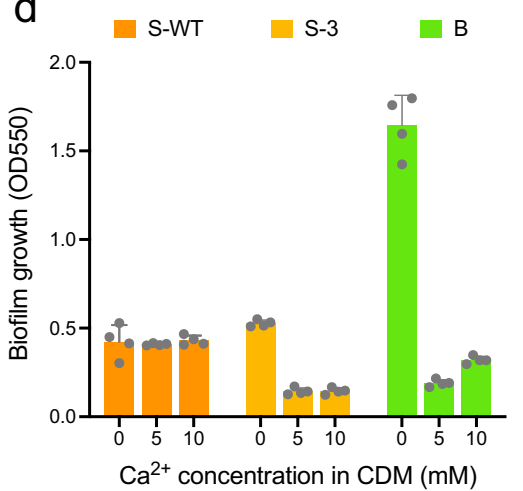

Fig. 6 Effects of mutations in the S. epidermidis biofilm-associated protein (Bap). a Motility assay of B. subtilis (B) to the presence of bacterial supernatants of overnight cultures of S. epidermidis (S): wild-type (S-WT), and mutants (S-1 and S-3). CDM was used as a control (C; left). Snapshots of colonies on CDM-agar plates incubated at $37^{\circ} \mathrm{C}$ for $24 \mathrm{~h}$. Scale bar, $1 \mathrm{~mm}$. b Identification of protein domains in the biofilmassociated protein Bap. Domain identification was performed with the InterPro ${ }^{71}$ and NCBI-CDD ${ }^{72}$ databases: MSCRAMM is the acronym for "microbial surface components recognizing adhesive matrix molecules", which are adhesin proteins that mediate their attachment to surfaces $^{73}$. Cadherin repeat-like motifs are putative $\mathrm{Ca}^{2+}$ binding domains ${ }^{74}$. Putative lg domains are repeats found in several haemagglutinins and other cell surface proteins ${ }^{75}$. Polycystin cation channel protein is a putative $\mathrm{Ca}^{2+}$-permeable nonselective cation channel ${ }^{76}$. Location of the mutations present in bap of S. epidermidis S-1 and S-3 are shown. c Qualitative comparison of bacterial cell attachment to polystyrene in a micro-titer plate. The strains B. subtilis (B), and S. epidermidis wild type (S-WT) and S-3 strains were incubated in CDM supplemented with varying concentrations of $\mathrm{Ca}^{2+}(0,5$, and $10 \mathrm{mM})$, at $37^{\circ} \mathrm{C}$ for $24 \mathrm{~h}$. The experiment was performed in quadruplicates, and representative images are shown. d, Quantitative measurements of bacterial cell attachment ( $x$-axis indicates biofilm growth) to polystyrene in a micro-titer plate. The strains B. subtilis (B), and S. epidermidis wild type (S-WT) and S-3 strains were incubated in CDM supplemented with varying concentrations of $\mathrm{Ca}^{2+}(0,5$, and $10 \mathrm{mM})$, at $37^{\circ} \mathrm{C}$ for $24 \mathrm{~h}$. Data are presented as mean \pm S.D. Error bars represent standard deviation (SD) of the mean values of four experiments.

platform. A total of 5 million paired-end reads $(150 \mathrm{bp})$ were generated. FastQC version $0.11 .5^{64}$ was used to examine the quality of the reads. Taxonomic assignment of reads was performed with Kraken $2.0 .7^{65}$. The Rapid Annotations using Subsystems Technology (RAST) server ${ }^{66}$ was used to annotate the genomes. Identification of mutations was performed with Breseq $^{67}$, using the $S$. epidermidis $\mathrm{JH}$ data as a reference sequence (JAAUOD000000000).

\section{Antimicrobial assay}

Each B. subtilis strain was inoculated in $100 \mathrm{~mL}$ of CDM and grown at $37^{\circ} \mathrm{C}$. Cells were harvested by centrifugation at $6000 \times g$ for $15 \mathrm{~min}$. The supernatants were transferred into a clean tube, filtered through nitrocellulose Whatman filters $(0.45$ and $0.2 \mu \mathrm{m})$ and stored at $4{ }^{\circ} \mathrm{C}$ for subsequent analysis. Antimicrobial activity of $150 \mu \mathrm{L}$ of the bacterial supernatants was assessed on CDM-agar plates, using S. epidermidis wildtype strain as indicator strain.

\section{Chlorotetain identification}

UHPLC-M analysis was performed using a Dionex Ultimate 3000 UHPLC chromatographic system combined with a Q Exactive mass spectrometer (both from Thermo Fisher Scientific) fitted with a heated electrospray source operated in the positive ion mode. UHPLC separation was performed on a Kinetex EVO C18, $2.6 \mu \mathrm{m}, 2.1 \mathrm{~mm} \times 150 \mathrm{~mm}$ column (Phenomenex, Maarsen, The Netherlands). The column was kept at $50 \pm$ $0.1{ }^{\circ} \mathrm{C}$ during analysis. Mobile phases were $\mathrm{A}: 100 \%$ water, $0.1 \%$ formic acid, and B: $100 \%$ ACN and $0.1 \%$ formic acid. The injection volume for all separations was $10 \mu \mathrm{L}$. Chromatographic elution was achieved under gradient conditions with a flow rate of $400 \mu \mathrm{L} / \mathrm{min}$. Elution started with an isocratic step of $1.0 \mathrm{~min}$ at $1 \% \mathrm{~B}$, followed by a linear gradient from 1 to $95 \%$ B (1.0-10.0 min. These conditions were maintained for $2 \mathrm{~min}$ before returning to $1 \% \mathrm{~B}$ in $0.03 \mathrm{~min}$ and equilibration at start conditions for $3 \mathrm{~min}$. The total runtime was $15 \mathrm{~min}$. The $\mathrm{Q}$ Exactive mass spectrometer was operated with a capillary voltage of $3.50 \mathrm{kV}$. The capillary temperature was set at $275^{\circ} \mathrm{C}$, and auxiliary gas temperature at $350^{\circ} \mathrm{C}$. The sheath gas 
B

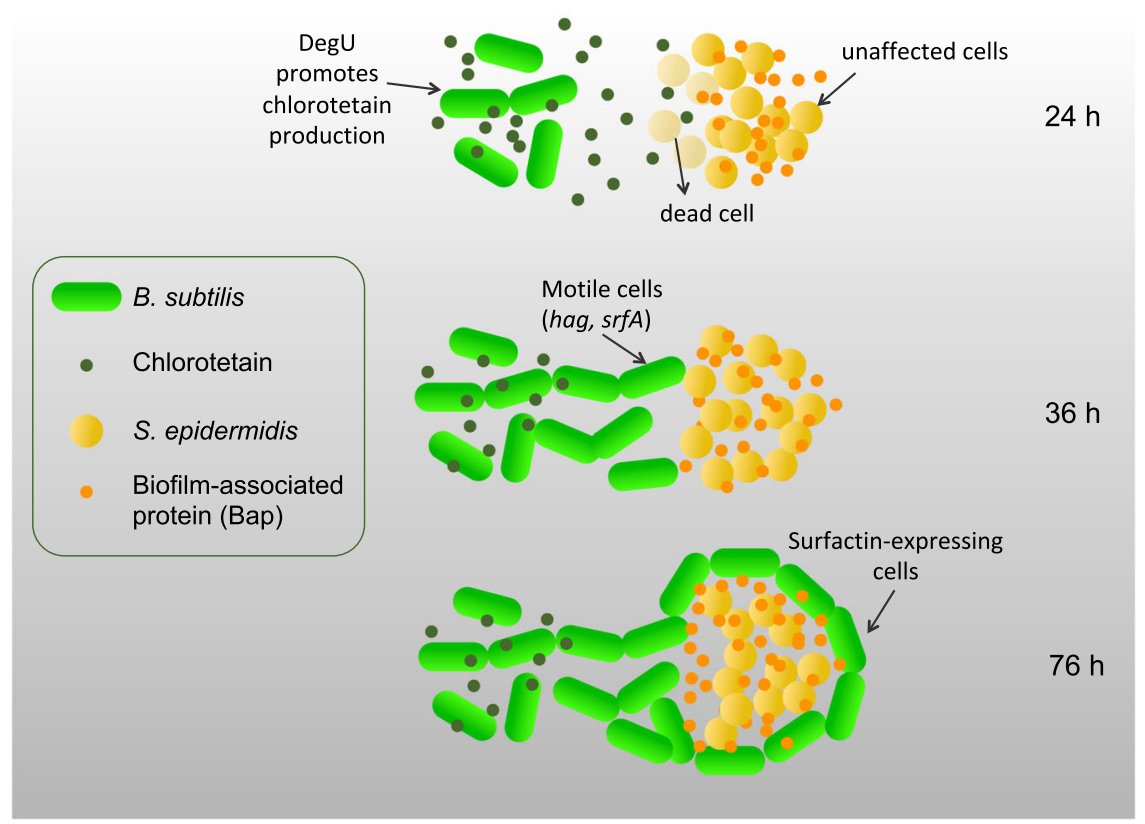

Fig. 7 Proposed model of interaction between S. epidermidis and B. subtilis. Bacterial colonies are able to interact at a close proximity. $B$. subtilis cells (rod-shaped bacteria in green) attack the neighbor colony of $S$. epidermidis (spherical-shaped bacteria in yellow) by producing chlorotetain (indicated with small dark green dots) during the first $24 \mathrm{~h}$ of incubation. S. epidermidis cells located in the diffusion zone of chlorotetain take the chlorotetain molecules up, and the release of L-anticapsin by chlorotetain hydrolyzation results in bacterial death ${ }^{41}$. However, cells in the periphery of the S. epidermidis colony remain unaffected, are able to develop the characteristic cell-to-cell attachment via biofilm-associated proteins (Bap; indicated with small orange dots). The Bap proteins require $\mathrm{Ca}^{2+}$ ions to acquire a proper folding and become functional, these proteins also mediate cell attachment to surfaces ${ }^{47,77}$. After 36 to $76 \mathrm{~h}$ incubation, the depletion of $\mathrm{Ca}^{2+}$ in the surroundings of the $S$. epidermidis colony triggers $B$. subtilis motility. A subpopulation of $B$. subtilis cells emerges, and utilizes sliding motility to reach the $S$. epidermidis colony. The $B$. subtilis engulf the remaining $S$. epidermidis colony, where the presence of Bap might facilitate the $B$. subtilis motility. Since high cell densities of $S$. epidermidis are resistant to chlorotetain, we speculate that the initial unaffected cells reach high cell densities and form biofilms structures, and thus, these cells tolerate the chlorotetain concentrations once the engulfment occurs.

pressure, auxiliary gas pressure and sweep gas flow rate were set at 50, 10, and one arbitrary units, respectively, with nitrogen gas. Spectra were recorded from 100 to $750 \mathrm{~m} / \mathrm{z}$ at a resolution of $70000 @ \mathrm{mz} 200$. Xcalibur 4.1 was used for data processing.

\section{Motility assay}

B. subtilis motility was assessed on CDM-agar plates. One fifty microliter of filtered $S$. epidermidis supernatants were located in the center of the CDMagar plates, and $5 \mu \mathrm{L}$ of $B$. subtilis was spotted on the plates around the $S$. epidermidis supernatant in a spiral configuration to evaluate motility at different diffusion distances. A plate with $150 \mu \mathrm{L}$ of CDM instead of bacterial supernatant was used as a control. CDM-agar plates were incubated $24 \mathrm{~h}$ at $37^{\circ} \mathrm{C}$.

\section{Disc assay}

Sterile $5.5 \mathrm{~mm}$ diameter filter paper discs were placed on CDM-agar plates. Two discs were used per plate. Twenty microliters of the bacterial supernatant or control medium (CDM) were placed on the center of the discs, and the plates were dried for $5 \mathrm{~min}$ by keeping them open in a laminar flow cabinet. Five microliters of an overnight culture of the $B$. subtilis strain was then inoculated at different distances to the edge of the discs. The plates were incubated at $37^{\circ} \mathrm{C}$ for $24 \mathrm{~h}$. After the incubation period, snapshots were taken with an imaging system ChemiDoc XRS (Bio-Rad).

\section{Intracellular and extracelular contents}

Each S. epidermidis strain (S-WT, S-1, S-3) was inoculated in $20 \mathrm{~mL}$ of CDM and grown overnight at $37^{\circ} \mathrm{C}$. Cells were harvested by centrifugation at $6000 \times g$ for $15 \mathrm{~min}$. The supernatants were transferred into a clean tube, filtered through nitrocellulose Whatman filters $(0.45$ and $0.2 \mu \mathrm{m})$ and stored at $4{ }^{\circ} \mathrm{C}$ for subsequent analysis. Motility activity of $B$. subtilis was tested with $150 \mu \mathrm{L}$ of the bacterial supernatants (extracellular) on CDMagar plates. For intracellular content, the bacterial pellet was resuspended in CDM (cell suspension) and sonicated on ice for 10 min using a VCX 130 Sonicator with cycle $10 \mathrm{~s}$ ON and $10 \mathrm{~s}$ OFF. Cell debris was removed by centrifugation at $6000 \times g$ for $15 \mathrm{~min}$ at $4{ }^{\circ} \mathrm{C}$. The supernatant was collected and filtered through a $0.45 \mu \mathrm{m}$ filter.

\section{Biofilm assays}

We employed a biofilm formation method using the dye crystal violet $(\mathrm{CV})^{68}$. Bacterial strains were grown overnight in CDM supplemented with different calcium concentrations (1, 5, and $10 \mathrm{mM})$. Grown was performed in $100 \mu \mathrm{L}$ per well in a 96-well plate. We used four replicates per treatment. The plate was incubated $26 \mathrm{~h}$ at $37^{\circ} \mathrm{C}$. After incubation, bacterial cultures were removed from the wells and rinsed two times with deionized water to remove unattached cells. Next, $125 \mu \mathrm{L}$ of a $0.1 \%$ solution of crystal violet in water to each well of the micro-titer plate was added. The micro-titer plate was incubated at room temperature for $15 \mathrm{~min}$. Then, it was rinsed three times with water, and dried by incubation at $37^{\circ} \mathrm{C}$ for $2 \mathrm{~h}$. For qualitative assays, the wells were photographed. Qualitative measurements were performed as follows. One twenty five microliter of $30 \%$ acetic acid in water to each well were added to solubilize the CV. The micro-titer plate was incubated at room temperature for $15 \mathrm{~min}$. One twenty five microliter of the solubilized CV was transferred to a new flat-bottomed micro-titer plate. Absorbance measurements were performed in a plate reader at $550 \mathrm{~nm}$ using $30 \%$ acetic acid in water as the blank.

\section{Plate-reader assays}

Cultures of $S$. epidermidis and $B$. subtilis were grown and prepared as described above. For growth measurements, cells were diluted 1:20 in CDM. The growth was recorded in $0.2 \mathrm{~mL}$ cultures in 96-well micro-titer 
plates and monitored by using a micro-titer plate reader VarioSkan (Thermo Fisher Scientific Inc., MA, USA). Growth was recorded with measurements of the optical density at $600 \mathrm{~nm}\left(\mathrm{OD}_{600}\right)$ every $10 \mathrm{~min}$ for $24 \mathrm{~h}$. Values were corrected for the background value of the corresponding medium used for growth.

\section{Flow cytometry}

B. subtilis strains were grown for interaction assays with S. epidermidis in CDM as described above. Biofilms were scraped from the CDM-agar surface and resuspended in $800 \mu \mathrm{L}$ PBS. The biofilms were disrupted by repetitive passes through a $23 \mathrm{G}$ needle as reported in a previous study ${ }^{69}$. Cells were subjected to a mild sonication to obtain a preparation of single cells as described ${ }^{70}$. A threshold for the FSC and SCC parameters was set (200 in both) in the FACS Canto flow cytometer (BD Biosciences, CA, USA) to remove all the events that do not correspond to cells. The GFP-signal at all the measured cells was recorded in 30,000 events and used for downstream analysis (named ungated events in the corresponding figures). GFP-signal measurements were obtained with a FACS Canto flow cytometer (BD Biosciences, CA, USA) using a $488 \mathrm{~nm}$ argon laser. Raw data was collected using the FACSDiva Software 5.0.3 (BD Biosciences). And the FlowJo software was used for data analysis (https://www.flowjo.com/).

\section{Statistics and reproducibility}

Statistical analyses were performed using Prism 6.01 (GraphPad software https://www.graphpad.com/). All experiments were repeated independently at least three times. All micrographs, including small insets, show representative images from three independent replicate experiments.

\section{Bioinformatics}

Alignments and sequences identities were determined by using BLAST ${ }^{62}$ and $\mathrm{RAST}^{66}$, using the full-length protein or DNA sequences. Protein domains were identified with the InterPro and NCDI-CDC databases. Identification of mutations was performed with breseq version $0.32 .1^{67}$.

\section{Reporting summary}

Further information on research design is available in the Nature Research Reporting Summary linked to this article.

\section{DATA AVAILABILITY}

The S. epidermidis $\mathrm{JH}, \mathrm{S}-1$ and S-3 genomes were submitted to NCBI and are publicly available in the following accession codes: JAAUOD000000000 (JH; WT), JABTXG000000000 (S-1) and JABTXF000000000 (S-3). Data supporting the findings of this work are available within the paper and its Supplementary Information files. All other data are available from the corresponding author on request.

Received: 22 April 2020; Accepted: 14 July 2020;

Published online: 06 August 2020

\section{REFERENCES}

1. Belkaid, Y. \& Segre, J. A. Dialogue between skin microbiota and immunity. Science 346, 954-959 (2014)

2. Cogen, A. L., Nizet, V. \& Gallo, R. L. Skin microbiota: a source of disease or defence? Br. J. Dermatol. 158, 442-455 (2008).

3. Kumar, A. \& Chordia, N. Role of microbes in human health. Appl. Microbiol. Open Access. 13, 1-3 (2017).

4. Radeck, J. et al. Anatomy of the bacitracin resistance network in Bacillus subtilis. Mol. Microbiol. 100, 607-620 (2016).

5. Grice, E. A. \& Segre, J. A. The skin microbiome. Nat. Rev. Microbiol. 16, 143-155 (2011).

6. Grice, E. A. The skin microbiome: potential for novel diagnostic and therapeutic approaches to cutaneous disease. Semin. Cutan. Med. Surg. 33, 98-103 (2014).

7. Gonzalez, D. J. et al. Microbial competition between Bacillus subtilis and Staphylococcus aureus monitored by imaging mass spectrometry. Microbiology 157, 2485-2492 (2011).

8. Hibbing, M. E., Fuqua, C., Parsek, M. R. \& Peterson, S. B. Bacterial competition: surviving and thriving in the microbial jungle. Nat. Rev. Microbiol. 8, 15-25 (2010).

9. Granato, E. T., Meiller-Legrand, T. A. \& Foster, K. R. The evolution and ecology of bacterial warfare. Curr. Biol. 29, R521-R537 (2019).
10. Stubbendieck, R. M. \& Straight, P. D. Multifaceted interfaces of bacterial competition. J. Bacteriol. 198, 2145-2155 (2016).

11. Hegarty, J. W., Guinane, C. M., Ross, R. P., Hill, C. \& Cotter, P. D. Bacteriocin production: a relatively unharnessed probiotic trait? F1000Research. 5, 2587 (2016).

12. Schluter, J., Nadell, C. D., Bassler, B. L. \& Foster, K. R. Adhesion as a weapon in microbial competition. ISME J. 9, 139-149 (2015).

13. Costa, O. Y. A., Raaijmakers, J. M. \& Kuramae, E. E. Microbial extracellular polymeric substances: ecological function and impact on soil aggregation. Front. Microbiol. 9, 1636 (2018).

14. Caulier, S. et al. Overview of the antimicrobial compounds produced by members of the Bacillus subtilis group. Front. Microbiol. 10, 302 (2019).

15. Serra, C. R., Earl, A. M., Barbosa, T. M., Kolter, R. \& Henriques, A. O. Sporulation during growth in a gut isolate of Bacillus subtilis. J. Bacteriol. 196, 4184-4196 (2014).

16. Petruk, G., Donadio, G., Lanzilli, M., Isticato, R. \& Monti, D. M. Alternative use of Bacillus subtilis spores: protection against environmental oxidative stress in human normal keratinocytes. Sci. Rep. 8, 1745 (2018).

17. Wilson, M. The skin and its indigenous microbiota. In Microbial Inhabitants of Humans: Their Ecology and Role in Health and Disease. 51-106 (Cambridge University Press, Cambridge, 2004).

18. Ara, K. et al. Foot odor due to microbial metabolism and its control. Can. J. Microbiol. 52, 357-364 (2006).

19. Otto, M. Staphylococcus epidermidis-the 'accidental' pathogen. Nat. Rev. Microbiol. 7, 555-567 (2009).

20. Schoenfelder, S. M. K. et al. Success through diversity-how Staphylococcus epidermidis establishes as a nosocomial pathogen. Int. J. Med. Microbiol. 300, 380-386 (2010)

21. Oliveira, N. M. et al. Biofilm formation as a response to ecological competition. PLoS Biol. 13, e1002191 (2015)

22. Lopez, D., Vlamakis, H. \& Kolter, R. Generation of multiple cell types in Bacillus subtilis. FEMS Microbiol. Rev. 33, 152-163 (2009).

23. Calvio, C. et al. Swarming differentiation and swimming motility in Bacillus subtilis are controlled by swrA, a newly identified dicistronic operon. J. Bacteriol. 187, 5356-5366 (2005).

24. van Gestel, J., Vlamakis, H. \& Kolter, R. From cell differentiation to cell collectives: Bacillus subtilis uses division of labor to migrate. PLOS Biol. 13, e1002141 (2015).

25. Kinsinger, R. F., Shirk, M. C. \& Fall, R. Rapid surface motility in Bacillus subtilis is dependent on extracellular surfactin and potassium ion. J. Bacteriol. 185, 5627-5631 (2003).

26. Kinsinger, R. F., Kearns, D. B., Hale, M. \& Fall, R. Genetic requirements for potassium ion-dependent colony spreading in Bacillus subtilis. J. Bacteriol. 187, 8462-8469 (2005).

27. Ogran, A. et al. The plant host induces antibiotic production to select the mostbeneficial colonizers. Appl. Environ. Microbiol. 85, e00512-e00519 (2019).

28. Lewis, K. Platforms for antibiotic discovery. Nat. Rev. Drug Discov. 12, 371-387 (2013).

29. Stubbendieck, R. M. \& Straight, P. D. Escape from lethal bacterial competition through coupled activation of antibiotic resistance and a mobilized subpopulation. PLoS Genet. 11, e1005722 (2015).

30. Özcengiz, G. \& Öğülür, I. Biochemistry, genetics and regulation of bacilysin biosynthesis and its significance more than an antibiotic. N. Biotechnol. 32, 612-619 (2015).

31. Rosenberg, G. et al. Not so simple, not so subtle: the interspecies competition between Bacillus simplex and Bacillus subtilis and its impact on the evolution of biofilms. npj Biofilms Microbiomes. 2, 15027 (2016).

32. Kearns, D. B. A field guide to bacterial swarming motility. Nat. Rev. Microbiol. 8, 634-644 (2010).

33. Veening, J. W. et al. Transient heterogeneity in extracellular protease production by Bacillus subtilis. Mol. Syst. Biol. 4, 184 (2008).

34. Amati, G., Bisicchia, P. \& Galizzi, A. DegU-P represses expression of the motility flache operon in Bacillus subtilis. J. Bacteriol. 186, 6003-6014 (2004).

35. Verhamme, D. T., Murray, E. J. \& Stanley-Wall, N. R. DegU and Spo0A jointly control transcription of two loci required for complex colony development by Bacillus subtilis. J. Bacteriol. 191, 100-108 (2009).

36. Marlow, V. L. et al. Phosphorylated DegU manipulates cell fate differentiation in the Bacillus subtilis biofilm. J. Bacteriol. 196, 16-27 (2014).

37. Newman, J. A., Rodrigues, C. \& Lewis, R. J. Molecular basis of the activity of SinR Protein, the master regulator of biofilm formation in Bacillus subtilis. J. Biol. Chem. 288, 10766-10778 (2013).

38. Vlamakis, H., Chai, Y., Beauregard, P., Losick, R. \& Kolter, R. Sticking together: building a biofilm the Bacillus subtilis way. Nat. Rev. Microbiol. 11, 157-168 (2013).

39. Kearns, D. B., Chu, F., Branda, S. S., Kolter, R. \& Losick, R. A master regulator for biofilm formation by Bacillus subtilis. Mol. Microbiol. 55, 739-749 (2005). 
40. Wu, S. C. et al. Functional production and characterization of a fibrin-specific single-chain antibody fragment from Bacillus subtilis: Effects of molecular chaperones and a wall-bound protease on antibody fragment production. Appl. Environ. Microbiol. 68, 3261-3269 (2002).

41. Kenig, M. \& Abraham, E. P. Antimicrobial activities and antagonists of bacilysin and anticapsin. J. Gen. Microbiol. 94, 37-45 (1976).

42. Ertekin, O. et al. Analysis of a bac operon-silenced strain suggests pleiotropic effects of bacilysin in Bacillus subtilis. J. Microbiol. 58, 297-313 (2020).

43. Mariappan, A., Makarewicz, O., Chen, X. H. \& Borriss, R. Two-component response regulator DegU controls the expression of bacilysin in plant-growth-promoting bacterium Bacillus amyloliquefaciens FZB42. J. Mol. Microbiol. Biotechnol. 22, 114-125 (2012).

44. Verhamme, D. T., Kiley, T. B. \& Stanley-Wall, N. R. DegU co-ordinates multicellular behaviour exhibited by Bacillus subtilis. Mol. Microbiol. 65, 554-568 (2007).

45. Rajavel, M., Mitra, A. \& Gopal, B. Role of Bacillus subtilis BacB in the synthesis of bacilysin. J. Biol. Chem. 284, 31882-31892 (2009).

46. Dahl, M. K., Msadek, T., Kunst, F. \& Rapoport, G. Mutational analysis of the Bacillus subtilis DegU regulator and its phosphorylation by the DegS protein kinase. J. Bacteriol. 173, 2539-2347 (1991).

47. Lasa, I. \& Penadés, J. R. Bap: a family of surface proteins involved in biofilm formation. Res. Microbiol. 157, 99-107 (2006).

48. Perez, T. D. \& Nelson, W. J. Cadherin adhesion: mechanisms and molecular interactions. Handb. Exp. Pharmacol. 165, 3-21 (2004).

49. Mhatre, E. et al. Presence of calcium lowers the expansion of Bacillus subtilis colony biofilms. Microorganisms 5, 7 (2017).

50. Chiller, K., Selkin, B. A. \& Murakawa, G. J. Skin microflora and bacterial infections of the skin. J. Investig. Dermatol. Symp. Proc. 6, 170-174 (2001).

51. Claudel, J. P. et al. Staphylococcus epidermidis: a potential new player in the physiopathology of acne? Dermatology 235, 287-294 (2019).

52. Hilton, M. D., Alaeddinoglu, N. G. \& Demain, A. L. Bacillus subtilis mutant deficient in the ability to produce the dipeptide antibiotic bacilysin: isolation and mapping of the mutation. J. Bacteriol. 170, 1018-1020 (1988).

53. Steinberg, N. et al. The extracellular matrix protein TasA is a developmental cue that maintains a motile subpopulation within Bacillus subtilis biofilms. Sci. Signal. 13, eaaw8905 (2020).

54. Gallegos-Monterrosa, R. et al. Lysinibacillus fusiformis M5 induces increased complexity in Bacillus subtilis 168 colony biofilms via hypoxanthine. J. Bacteriol. 199, e00204-e00217 (2017).

55. Tecon, R., Ebrahimi, A., Kleyer, H., Levi, S. E. \& Or, D. Cell-to-cell bacterial interactions promoted by drier conditions on soil surfaces. Proc. Natl Acad. Sci. USA 115, 9791-9796 (2018).

56. Rosier, R. L. \& Langkilde, T. Behavior under risk: how animals avoid becoming dinner. Nat. Educ. Knowl. 2, 8 (2011).

57. Arnott, G. \& Elwood, R. W. Assessment of fighting ability in animal contests. Anim. Behav. 77, 991-1004 (2009).

58. Johnson, D. D. P. \& Toft, M. D. Grounds for war: the evolution of territorial conflict. Int. Secur. 38, 7-38 (2014).

59. Zhao, X. \& Kuipers, O. P. Identification and classification of known and putative antimicrobial compounds produced by a wide variety of Bacillales species. BMC Genomics. 17, 882 (2016)

60. Landy, M., Warren, G. H., Rosenmanm, S. B. \& Colio, L. G. Bacillomycin: an antibiotic from Bacillus subtilis active against pathogenic fungi. Proc. Soc. Exp. Biol. Med. 67, 539-541 (1948).

61. Li, X., Yuan, C., Xing, L. \& Humbert, P. Topographical diversity of common skin microflora and its association with skin environment type: An observational study in Chinese women. Sci. Rep. 7, 18046 (2017).

62. Madden, T. The BLAST Sequence Analysis Tool. 2013 Mar 15. In: The NCB Handbook [Internet]. 2nd edn. (National Center for Biotechnology Information (US), Bethesda, 2013).

63. Goel, A., Santos, F., de Vos, W. M., Teusink, B. \& Molenaar, D. Standardized assay medium to measure Lactococcus lactis enzyme activities while mimicking intracellular conditions. Appl. Environ. Microbiol. 78, 134-143 (2012).

64. Andrews, S. FastQC: a quality control tool for high throughput sequence data. http://www.bioinformatics.babraham.ac.uk/projects/fastqc (2010).

65. Wood, D. E. \& Salzberg, S. L. Kraken: ultrafast metagenomic sequence classification using exact alignments. Genome Biol. 15, R46 (2014).

66. Aziz, R. K. et al. The RAST Server: rapid annotations using subsystems technology. BMC Genom. 9, 75 (2008).

67. Deatherage, D. E. \& Barrick, J. E. Identification of mutations in laboratory-evolved microbes from next-generation sequencing data using breseq. Methods Mol. Biol. 1151, 165-188 (2014).
68. O'Toole, G. A. Microtiter dish biofilm formation assay. J. Vis. Exp. 47, 1-2 (2010).

69. Vlamakis, H., Aguilar, C., Losick, R. \& Kolter, R. Control of cell fate by the formation of an architecturally complex bacterial community. Genes Dev. 22, 945-953 (2008).

70. Branda, S. S., Chu, F., Kearns, D. B., Losick, R. \& Kolter, R. A major protein component of the Bacillus subtilis biofilm matrix. Mol. Microbiol. 49, 1229-1328 (2006).

71. Hunter, S. et al. InterPro: the integrative protein signature database. Nucleic Acids Res. 37, D211-D215 (2009).

72. Marchler-Bauer, A. et al. CDD/SPARCLE: functional classification of proteins via subfamily domain architectures. Nucleic Acids Res. 45, D200-D203 (2017).

73. Foster, T. J. The MSCRAMM family of cell-wall-anchored surface proteins of grampositive Cocci. Trends Microbiol. 27, 927-941 (2019).

74. Sotomayor, M. \& Schulten, K. The allosteric role of the $\mathrm{Ca}_{2}^{+}$switch in adhesion and elasticity of C-cadherin. Biophys. J. 94, 4621-4633 (2008).

75. Bentley, S. D. et al. Sequencing and analysis of the genome of the Whipple's disease bacterium Tropheryma whipplei. Lancet 361, 637-644 (2003).

76. Chen, X. Z. et al. Polycystin-L is a calcium-regulated cation channel permeable to calcium ions. Nature 401, 383-386 (1999).

77. Cucarella, C. et al. Bap, a Staphylococcus aureus surface protein involved in biofilm formation. J. Bacteriol. 183, 2888-2896 (2001).

\section{ACKNOWLEDGEMENTS}

We thank Anne de Jong (Department of Molecular Genetics, University of Groningen) for his help with sequences analysis. J.A.H.V. and O.P.K. were financed by the Netherlands Organization for Scientific Research (NWO), research program TTW (13858). L.Z. was financially supported by the China Scholarship Council (201606910037).

\section{AUTHOR CONTRIBUTIONS}

J.A.H.V. conceived the study. J.A.H.V. designed and carried out all the experiments. L. Z. performed sample preparations for UHPLC analysis and interpreted data. M.d.V. performed the UHPLC-M analyses and analyzed the data. J.A.H.V. and O.P.K. wrote the manuscript. O.P.K. provided supervision. All authors discussed the results and commented on the manuscript.

\section{COMPETING INTERESTS}

The authors declare no competing interests.

\section{ADDITIONAL INFORMATION}

Supplementary information is available for this paper at https://doi.org/10.1038/ s41522-020-00140-0.

Correspondence and requests for materials should be addressed to O.P.K.

Reprints and permission information is available at http://www.nature.com/ reprints

Publisher's note Springer Nature remains neutral with regard to jurisdictional claims in published maps and institutional affiliations.

Access This article is licensed under Attribution 4.0 International License, which permits use, sharing, adaptation, distribution and reproduction in any medium or format, as long as you give appropriate credit to the original author(s) and the source, provide a link to the Creative Commons license, and indicate if changes were made. The images or other third party material in this article are included in the article's Creative Commons license, unless indicated otherwise in a credit line to the material. If material is not included in the article's Creative Commons license and your intended use is not permitted by statutory regulation or exceeds the permitted use, you will need to obtain permission directly from the copyright holder. To view a copy of this license, visit http://creativecommons. org/licenses/by/4.0/.

(c) The Author(s) 2020 